\title{
THE BLOCH-KATO CONJECTURE AND A THEOREM OF SUSLIN-VOEVODSKY
}

\author{
THOMAS GEISSER AND MARC LEVINE
}

\begin{abstract}
We give a new proof of the theorem of Suslin-Voevodsky which shows that the Bloch-Kato conjecture implies a portion of the BeilinsonLichtenbaum conjectures. Our proof does not rely on resolution of singularities, and thereby extends the Suslin-Voevodsky to positive characteristic.
\end{abstract}

\section{INTRODUCTION}

The purpose of this paper is to give an alternate proof of the main result of [19], along the lines of [10]; our proof does not rely on resolution of singularities, hence extends the results of [19] to varieties over fields of arbitrary characteristic. The new ingredient which enables us to apply the techniques of [10] to motivic cohomology is the surjectivity result of [7], Remark following Corollary 4.3.

Let $F$ be a field, $q \geq 0$ an integer, and $m>1$ an integer prime to the characteristic of $F$. We have the Milnor $K$-group $K_{n}^{M}(F)$, and the Galois symbol

$$
\vartheta_{q, F}: K_{q}^{M}(F) / m \rightarrow H_{\text {ét }}^{q}\left(F, \mu_{m}^{\otimes q}\right) .
$$

The Bloch-Kato conjecture [3] asserts that the map $\vartheta_{q, F}$ is an isomorphism for all $F, q$ and $m$.

As described in $\S 2.2$, we will use Bloch's higher Chow groups as our definition of motivic cohomology for smooth quasi-projective $k$-schemes, and use the Mayer-Vietoris property of motivic cohomology to extend to schemes which are a localization of a smooth scheme of finite type over $k$ (we call such a scheme essentially smooth over $k$ ). We use Bloch's cycle complexes to define the weight $q$ motivic complex $\Gamma(q)_{X}$, as an object in the derived category of Zariski sheaves on an essentially smooth $k$-scheme $X$.

For an essentially smooth scheme $X$ over a field $k$ of characteristic prime to $m$, let $\epsilon: X_{\text {ét }} \rightarrow X_{\text {Zar }}$ be the change of topology map. We construct in $\S 3.9$ a natural cycle class map (in the derived category)

$$
\operatorname{cl}_{X}^{a}: \Gamma(a)_{X} \otimes^{L} \mathbb{Z} / m \rightarrow \tau_{\leq a} R \epsilon_{*}\left(\mu_{m}^{\otimes a}\right) .
$$

Our main result is

Theorem 1.1. Let $k$ be a field, and fix an integer $m$ prime to the characteristic of $k$. Suppose that the maps $\vartheta_{q, F}: K_{q}^{M}(F) / m \rightarrow H_{\mathrm{èt}}^{q}\left(F, \mu_{m}^{\otimes q}\right)$ are surjective for all finitely generated field extensions $F$ of $k$. Then the cycle class map (1.2) is a quasi-isomorphism for all essentially smooth $X$ over $k$ and all $a$ with $0 \leq a \leq q$.

1991 Mathematics Subject Classification. Primary 19E20; Secondary 19D45, 19E08, 14 C25.

Key words and phrases. Motivic cohomology, étale cohomology, Milnor $K$-theory, higher Chow groups.

The second author is supported by the NSF. 
In particular, under the assumption in Theorem 1.1, the map

$$
\mathrm{cl}^{q}: H^{p}(R, \mathbb{Z} / m(q)) \rightarrow H_{\text {ét }}^{p}\left(R, \mu_{m}^{\otimes q}\right)
$$

is an isomorphism for all smooth local $k$-algbras $R$, essentially of finite type over $k$, and for all $p \leq q$.

The conclusion of Theorem 1.1 is a part of the Beilinson-Lichtenbaum conjectures (see e.g. [12]).

Using Voevodsky's result [21] that the Bloch-Kato conjecture is true for $m$ a power of 2, we may apply Theorem 1.1 to extend the consequences of [21] to characteristic $p>0$

Corollary 1.2. For $m=2^{\nu}$, the cycle class map (1.2) is a quasi-isomorphism for all $X$ essentially smooth over $k$.

Similarly, the Merkerjev-Suslin theorem [13] (which is the Bloch-Kato conjecture for $q=2$ ) gives as in [19]

Corollary 1.3. Let $k$ be a field, and let $m$ be prime to char $k$. Then the cycle class map (1.2) is a quasi-isomorphism for all $a, 0 \leq a \leq 2$, and all $X$ essentially smooth over $k$.

\section{Prelimiaries}

We recall some of the notations and results from [7] for the reader's convenience. We fix a field $k$.

2.1. Cycle complexes. Let $\Delta^{n}$ be the affine space

$$
\Delta^{n}:=\operatorname{Spec} k\left[t_{0}, \ldots, t_{n}\right] / \sum_{i=0}^{n} t_{i}-1 ;
$$

sending $n$ to $\Delta^{n}$ extends to form the cosimplicial $k$-scheme $\Delta^{*}$. A subscheme of $\Delta^{m}$ defined by equations of the form $t_{i_{1}}=\ldots=t_{i_{r}}=0$ is called a face of $\Delta^{n}$ (we consider $\Delta^{n}$ a face of $\Delta^{n}$ as well).

Let $X$ be a quasi-projective $k$-scheme. Bloch [1] has defined the cycle complex $z^{q}(X, *)$, with $z^{q}(X, p)$ the subgroup of the group $z^{q}\left(X \times \Delta^{n}\right)$ of codimension $q$ cycles on $X \times \Delta^{p}$ generated by codimension $q$ subvarieties $W$ of $X \times \Delta^{p}$ such that, for each face $F$ of $\Delta^{p}, W \cap X \times F$ has codimension $\geq q$ on $X \times F$. For each order-preserving map

$$
g:\{0<\ldots<n\} \rightarrow\{0<\ldots<m\}
$$

and each $W \in z^{q}(X, m)$, the pull-back $(\mathrm{id} \times g)^{*}(W)$ via the induced map id $\times g$ : $X \times \Delta^{n} \rightarrow X \times \Delta^{m}$ is defined and is in $z^{q}(X, n)$. Thus, sending $m$ to $z^{q}(X, m)$ gives rise to a simplicial abelian group, and $z^{q}(X, *)$ is defined as the associated chain complex. The higher Chow groups of $X, \mathrm{CH}^{q}(X, p)$, are then defined as the homology

$$
\mathrm{CH}^{q}(X, p):=H_{p}\left(z^{q}(X, *)\right) .
$$

Let $\mathcal{S}$ be a finite set of closed subschemes of $X$, including $X$ as an element. We have the subcomplex $z^{q}(X, *)_{\mathcal{S}}$ of $z^{q}(X, *)$ with $z^{q}(X, p)_{\mathcal{S}}$ being the subgroup of $z^{q}(X, p)$ generated by subvarieties $W$ which intersect $S \times F$ properly on $X \times \Delta^{p}$, for all $S$ in $\mathcal{S}$, and all faces $F$ of $\Delta^{p}$. If $Y$ is in $\mathcal{S}$, and $\mathcal{S}(Y)$ is the subset of $\mathcal{S}$ consisting of those $S$ contained in $Y$, we write $z^{q}(Y, *)_{\mathcal{S}}$ for $z^{q}(Y, *)_{\mathcal{S}(Y)}$. By definition, the 
operation of intersection with $Y \times \Delta^{p}$ defines pull-back maps $i_{Y}^{*}: z^{q}(X, p)_{\mathcal{S}} \rightarrow$ $z^{q}(Y, p)_{\mathcal{S}}$, giving a map of complexes $i_{Y}^{*}: z^{q}(X, *)_{\mathcal{S}} \rightarrow z^{q}(Y, *)_{\mathcal{S}}$. If $X$ is smooth over $k$ and affine, then the proof of [11, Chap. II, Theorem 3.5.14], shows that the inclusion

$$
z^{q}(X, *)_{\mathcal{S}} \rightarrow z^{q}(X, *)
$$

is a quasi-isomorphism, giving a functorial pull-back map

$$
i_{Y}^{*}: \mathrm{CH}^{q}(X, *) \rightarrow \mathrm{CH}^{q}(Y, *) .
$$

The localization property of the higher Chow groups [2] (see $\S 2.3$ below for more details) implies that, if $\mathcal{U}:=\left\{U_{1}, \ldots, U_{n}\right\}$ is an open cover of a smooth quasiprojective $k$-scheme $X$, then the total Cech complex $z^{q}(\mathcal{U}, *)$ for $z^{q}(-, *)$ with respect to the cover $\mathcal{U}$ is quasi-isomorphic to $z^{q}(X, *)$ via the augmentation. This implies that, for $X$ smooth over $k$, we may construct a well-defined object $z^{q}(X, *)$ in the derived category $\mathbf{D}^{-}(\mathbf{A b})$ by taking the Čech complex $z^{q}(\mathcal{U}, *)$ associated to an open cover $\mathcal{U}$ with the $U_{i}$ quasi-projective over $k$. If $X^{\prime}$ is a localization of a smooth $k$-scheme $X$, we may define $z^{q}\left(X^{\prime}, *\right)$ by taking the inductive limit of complexes $z^{q}\left(X^{\prime \prime}, *\right)$, over $X^{\prime} \subset X^{\prime \prime} \subset X$, with $X^{\prime \prime}$ open in $X$, where we fix from the start a quasi-projective open cover of $X$. This enables us to extend the definition of $\mathrm{CH}^{q}(X, p)$ to schemes essentially smooth over $k$, and the various results described above continue to hold. In particular, all the notions described above make sense for a $k$-scheme $X^{\prime}$ of the form $\operatorname{Spec}\left(\mathcal{O}_{X, v}\right)$, where $\mathcal{O}_{X, v}$ is the local ring of a smooth quasi-projective variety $X$ at a finite set $v$ of closed subvarieties.

2.2. Motivic cohomology. The higher Chow groups have been incorporated into a general theory of motivic cohomology via the categorical constructions of Voevodsky [20] and the second author [11]. In case resolution of singularities holds for finite type $k$-schemes (e.g., $k$ has characteristic zero), the motivic cohomology of [20] and [11] are canonically isomorphic; for smooth quasi-projective $X$, both theories agree with the higher Chow groups via a canonical isomorphism

$$
\mathrm{CH}^{q}(X, p) \cong H^{2 q-p}(X, \mathbb{Z}(q)) .
$$

Even without assuming resolution of singularities, the isomorphism (2.2) holds for the motivic cohomology of [11] (see [11, Chap. II, Theorem 3.6.6]).

We reindex Bloch's cycle complex to reflect the isomorphism (2.2), defining the cohomological cycle complex $\mathcal{Z}^{q}(X, *)$, for smooth quasi-projective $X$, by

$$
\mathcal{Z}^{q}(X, p):=z^{q}(X, 2 q-p),
$$

and take the cohomology $H^{p}\left(\mathcal{Z}^{q}(X, *)\right)$ as the definition of the motivic cohomology $H^{p}(X, \mathbb{Z}(q))$. We define the subcomplex $\mathcal{Z}^{q}(X, p)_{\mathcal{S}}$ similarly.

Let $m$ be an integer, and $X$ smooth and quasi-projective. The mod $m$ motivic cohomology, $H^{p}(X, \mathbb{Z} / m(q))$, is defined by

$$
H^{p}(X, \mathbb{Z} / m(q)):=H^{p}\left(\mathcal{Z}^{q}(X, *) \otimes \mathbb{Z} / m\right) .
$$

The mod $m$ motivic cohomology has a natural definition via the motivic categories of [20] and [11] as well, which agrees with the definition given here, subject to the restrictions described above.

As in $\S 2.1$, we may extend the definition of $\mathcal{Z}^{q}(X, *)$ to $X$ essentially smooth over $k$, with the understanding that, if $X$ is not the localization of a quasi-projective scheme over $k, \mathcal{Z}^{q}(X, *)$ is only defined as an object in $\mathbf{D}^{-}(\mathbf{A b})$. Similar remarks 
hold for the mod $m$ complexes. This extends the definition of $H^{p}(X, \mathbb{Z}(q))$ and $H^{p}(X, \mathbb{Z} / m(q))$ to $X$ essentially smooth over $k$.

2.3. Localization. Let $W$ be a closed subset of a quasi-projective $k$-scheme $X$, with inclusion $i: W \rightarrow X$, and open complement $j: U \rightarrow X$. We set

$$
z_{W}^{q}(X, *):=\operatorname{cone}\left(j^{*}: z^{q}(X, *) \rightarrow z^{q}(U, *)\right) .
$$

Suppose now that $W$ has pure codimension $d$ on $X$, giving us the push-forward $i_{*}: z^{q-d}(W, *) \rightarrow z^{q}(X, *)$. Since $j^{*} \circ i_{*}=0$, we have the canonical map

$$
i_{*}^{W}: z^{q-d}(W, *) \rightarrow z_{W}^{q}(X, *),
$$

which is shown in [2] to be a quasi-isomorphism. Combining $i_{*}^{W}$ with the standard cone sequence gives us the distinguished triangle

$$
z^{q-d}(W, *) \stackrel{i_{*}}{\longrightarrow} z^{q}(X, *) \stackrel{j^{*}}{\longrightarrow} z^{q}(U, *) \rightarrow z^{q-d}(W, *-1) .
$$

If $X$ and $W$ are smooth, we have the cohomological distinguished triangle

$$
\mathcal{Z}^{q-d}(W, *)[-2 d] \stackrel{i_{*}}{\longrightarrow} \mathcal{Z}^{q}(X, *) \stackrel{j^{*}}{\longrightarrow} \mathcal{Z}^{q}(U, *) \rightarrow \mathcal{Z}^{q-d}(W, *)[-2 d+1],
$$

giving the localization sequence for motivic cohomology

$$
\begin{aligned}
\rightarrow H^{p-2 d}(W, \mathbb{Z}(q-d)) \stackrel{i_{*}}{\longrightarrow} H^{p}(X, \mathbb{Z}(q)) \stackrel{j^{*}}{\longrightarrow} H^{p}(U, \mathbb{Z}(q)) & \\
& \stackrel{\partial}{\longrightarrow} H^{p-2 d+1}(W, \mathbb{Z}(q-d)) \rightarrow
\end{aligned}
$$

The isomorphism

$$
i_{*}^{W}: H^{p-2 d}(W, \mathbb{Z}(q-d)) \rightarrow H^{p}(X, \mathbb{Z}(q))
$$

resulting from the quasi-isomorphism (2.3) is the so-called Gysin isomorphism for motivic cohomology.

2.4. Relative motivic cohomology. We describe a relative version of motivic cohomology; for simplicity, we restrict ourselves to the affine case.

Let $X$ be a smooth affine $k$-scheme, essentially of finite type over $k$, and let $Z_{1}, \ldots, Z_{n}$ be closed subschemes. For an index $I \subset\{1, \ldots, n\}$, let $Z_{I}:=\cap_{i \in I} Z_{i}$. We suppose that all the $Z_{I}$ are smooth over $k$.

Let $\mathcal{S}$ be a finite set of closed subsets of $X$, containing all the $Z_{I}$. Form the complex $\mathcal{Z}^{q}\left(X ; Z_{1}, \ldots, Z_{n}, *\right)_{\mathcal{S}}$ as the total complex of the double complex

$$
\mathcal{Z}^{q}(X, *)_{\mathcal{S}} \rightarrow \bigoplus_{i=1}^{n} \mathcal{Z}^{q}\left(Z_{i}, *\right)_{\mathcal{S}} \rightarrow \ldots \rightarrow \bigoplus_{\substack{I \subset\{1, \ldots, n\} \\|I|=j}} \mathcal{Z}^{q}\left(Z_{I}, *\right)_{\mathcal{S}} \rightarrow \ldots
$$

with the total degree of $\mathcal{Z}^{q}\left(Z_{I}, p\right)_{\mathcal{S}}$ being $p+|I|$. Here the differential

$$
\bigoplus_{\substack{I \subset\{1, \ldots, n\} \\|I|=j}} \mathcal{Z}^{q}\left(Z_{I}, *\right)_{\mathcal{S}} \rightarrow \bigoplus_{\substack{I \subset\{1, \ldots, n\} \\|I|=j+1}} \mathcal{Z}^{q}\left(Z_{I}, *\right)_{\mathcal{S}}
$$

is a signed sum of the pull-back maps

$$
\mathcal{Z}^{q}\left(Z_{I}, *\right)_{\mathcal{S}} \rightarrow \mathcal{Z}^{q}\left(Z_{I \cup\{i\}}, *\right)_{\mathcal{S}} ; \quad i \notin I,
$$

induced by the inclusions $Z_{I \cup\{i\}} \subset Z_{I}$; the sign is $(-1)^{l}$, where $l$ is the number of $j \in I$ with $j>i$. 
The complexes $\mathcal{Z}^{q}\left(X ; Z_{1}, \ldots, Z_{n}, *\right)_{\mathcal{S}}$ for varying $\mathcal{S}$ are all quasi-isomorphic, since the inclusion (2.1) is a quasi-isomorphism. We will often drop the $\mathcal{S}$ from the notation.

The relative motivic cohomology $H^{p}\left(X ; Z_{1}, \ldots, Z_{n}, \mathbb{Z}(q)\right)$ is defined as

$$
H^{p}\left(X ; Z_{1}, \ldots, Z_{n}, \mathbb{Z}(q)\right):=H^{p}\left(\mathcal{Z}^{q}\left(X ; Z_{1}, \ldots, Z_{n}, *\right)\right) ;
$$

the $\bmod m$ version is defined similarly by

$$
H^{p}\left(X ; Z_{1}, \ldots, Z_{n}, \mathbb{Z} / m(q)\right):=H^{p}\left(\mathcal{Z}^{q}\left(X ; Z_{1}, \ldots, Z_{n}, *\right) \otimes \mathbb{Z} / m\right) .
$$

Take $\mathcal{S}$ to contain all the $Z_{I}$. The pull-back maps $\mathcal{Z}^{q}\left(Z_{I}, *\right)_{\mathcal{S}} \rightarrow \mathcal{Z}^{q}\left(Z_{I \cup\{n\}}, *\right)_{\mathcal{S}}$ induce the map of complexes

$$
i_{n}^{*}: \mathcal{Z}^{q}\left(X ; Z_{1}, \ldots, Z_{n-1}, *\right)_{\mathcal{S}} \rightarrow \mathcal{Z}^{q}\left(Z_{n} ; Z_{1, n}, \ldots, Z_{n-1, n}, *\right)_{\mathcal{S}}
$$

and we have the evident isomorphism

$$
\mathcal{Z}^{q}\left(X ; Z_{1}, \ldots, Z_{n}, *\right)_{\mathcal{S}} \cong \operatorname{cone}\left(i_{n}^{*}\right)[-1] .
$$

This gives us the long exact relativization sequence

$$
\begin{aligned}
\rightarrow & H^{p-1}\left(Z_{n} ; Z_{1, n}, \ldots, Z_{n-1, n}, \mathbb{Z}(q)\right) \rightarrow H^{p}\left(X ; Z_{1}, \ldots, Z_{n}, \mathbb{Z}(q)\right) \\
& \rightarrow H^{p}\left(X ; Z_{1}, \ldots, Z_{n-1}, \mathbb{Z}(q)\right) \rightarrow H^{p}\left(Z_{n} ; Z_{1, n}, \ldots, Z_{n-1, n}, \mathbb{Z}(q)\right) \rightarrow
\end{aligned}
$$

and similarly for the mod $m$ version.

2.5. Normal crossing schemes. We describe an extension of the cycle complexes $\mathcal{Z}^{q}(X, *)$ to the simplest type of singular schemes, those formed by a union of normal crossing subschemes. Taking the component in degree $2 q$ gives an extension to normal crossing schemes of the notion of a codimension $q$ cycle on a smooth variety.

Let $X$ be an essentially smooth $k$-scheme, $Y_{1}, \ldots, Y_{n}$ closed subschemes such that each intersection $Y_{I}$ is essentially smooth. Let $Y$ be the union of the $Y_{i}$. We call such a $k$-scheme a normal crossing scheme. Let $\mathcal{S}$ be a finite set of closed subsets of $Y$, containing all the $Y_{I}$, and let $\mathcal{S}_{I} \subset \mathcal{S}$ be the set of those closed subsets contained in $Y_{I}$. We assume that for $S \in \mathcal{S}$, each irreducible component of $S \cap Y_{I}$ is also in $\mathcal{S}$; we write $\mathcal{Z}^{q}\left(Y_{I}, *\right)_{\mathcal{S}}$ for $\mathcal{Z}^{q}\left(Y_{I}, *\right)_{\mathcal{S}_{I}}$.

Let $\mathcal{Z}^{q}(Y, *)_{\mathcal{S}}$ be the complex defined by the term-wise exactness of

$$
0 \rightarrow \mathcal{Z}^{q}(Y, *)_{\mathcal{S}} \rightarrow \bigoplus_{i=1}^{n} \mathcal{Z}^{q}\left(Y_{i}, *\right)_{\mathcal{S}} \stackrel{\alpha}{\longrightarrow} \bigoplus_{1 \leq i<j \leq n}^{n} \mathcal{Z}^{q}\left(Y_{i, j}, *\right)_{\mathcal{S}}
$$

where $\alpha$ is the sum of the maps

$$
\mathcal{Z}^{q}\left(Y_{i}, *\right)_{\mathcal{S}} \rightarrow \mathcal{Z}^{q}\left(Y_{i, j}, *\right)_{\mathcal{S}}
$$

these in turn being the restriction map for $i<j$, and minus the restriction map for $i>j$. If we take $\mathcal{S}$ to be the set of irreducible components of all the $Y_{I}$, we write $\mathcal{Z}^{q}(Y, *)$ for $\mathcal{Z}^{q}(Y, *)_{\mathcal{S}}$. We write $z^{q}(Y)_{\mathcal{S}}$ for $\mathcal{Z}^{q}(Y, 2 q)_{\mathcal{S}}$ (which is just $\left.z^{q}(Y, 0)_{\mathcal{S}}\right)$.

Let $f: Y \rightarrow Y^{\prime}$ be a morphism of normal crossing schemes, and take $\mathcal{S}$ and $\mathcal{S}^{\prime}$ to be the minimal choices, i.e., $\mathcal{S}$ is the set of irreducible components of all the $Y_{I}$, and similarly for $\mathcal{S}^{\prime}$. Assume that the restriction of $f$ to each $Y_{I}$ factors as

$$
Y_{I} \stackrel{g}{\longrightarrow} Y_{J}^{\prime} \stackrel{i_{J}}{\longrightarrow} Y^{\prime}
$$


for some index $J$ (depending on $I$ ), with $g$ flat, and $i_{J}$ the inclusion. For each $i$, choose an index $j(i)$ such that $f\left(Y_{i}\right) \subset Y_{j(i)}^{\prime}$. Then each of the pull-back maps $f_{\mid Y_{j(i)}}^{*}: \mathcal{Z}^{q}\left(Y_{j(i)}^{\prime}, *\right)_{\mathcal{S}^{\prime}} \rightarrow \mathcal{Z}^{q}\left(Y_{i}, *\right)_{\mathcal{S}}$ is defined. Composing $f_{\mid Y_{j(i)}}^{*}$ with the projection

$$
\pi_{i}: \bigoplus_{j} \mathcal{Z}^{q}\left(Y_{j}^{\prime}, *\right)_{\mathcal{S}^{\prime}} \rightarrow \mathcal{Z}^{q}\left(Y_{j(i)}^{\prime}, *\right)_{\mathcal{S}^{\prime}}
$$

gives the map

$$
f_{i}^{*}: \bigoplus_{j} \mathcal{Z}^{q}\left(Y_{j}^{\prime}, *\right)_{\mathcal{S}^{\prime}} \rightarrow \mathcal{Z}^{q}\left(Y_{i}, *\right)_{\mathcal{S}^{\prime}}
$$

The sum of $f_{i}$ then gives a well-defined pull-back map

$$
f^{*}:=\sum_{i} f_{i}^{*}: \mathcal{Z}^{q}\left(Y^{\prime}, *\right) \rightarrow \mathcal{Z}^{q}(Y, *)
$$

independent of the choice of the $j(i)$. This extends the assignment $Y \mapsto \mathcal{Z}^{q}(Y, *)$ to a functor on the category of normal crossing $k$-schemes, with morphisms admitting a factorization as above.

If we enlarge the subset $\mathcal{S}$, we get an expanded functoriality, but it is not clear that the inclusion $\mathcal{Z}^{q}(Y, *)_{\mathcal{S}} \rightarrow \mathcal{Z}^{q}(Y, *)$ is a quasi-isomorphism. The complexes $\mathcal{Z}^{q}(Y, *)_{\mathcal{S}}$ for normal crossing $Y$ will play only a technical and auxiliary role in our argument, and we will never require that the cohomology $H^{*}\left(\mathcal{Z}^{q}(Y, *)_{\mathcal{S}}\right)$ is independent of the choice of $\mathcal{S}$.

If $Z_{1}, \ldots, Z_{m}$ are normal crossing subschemes of $Y$, and if $\mathcal{S}$ is chosen appropriately, we will have the relative cycle complex $\mathcal{Z}^{q}\left(Y ; Z_{1} \ldots, Z_{n}, *\right)_{\mathcal{S}}$ defined as in $\S 2.4$ for the smooth case.

We define a relative version $\mathcal{Z}^{q}(Y ; T, *)_{\mathcal{S}}^{\mathrm{ker}}$, where $T$ is a subset of $\left\{Y_{1}, \ldots, Y_{n}\right\}$, by taking the kernel of the projection

$$
\mathcal{Z}^{q}(Y, *)_{\mathcal{S}} \rightarrow \bigoplus_{Y_{i} \in T} \mathcal{Z}^{q}\left(Y_{i}, *\right)_{\mathcal{S}}
$$

The complexes $\mathcal{Z}^{q}(Y ; T, *)^{\text {ker }}$ have the functoriality similar to $\mathcal{Z}^{q}(Y, *)$, with the obvious additional condition that, in order for $f^{*}: \mathcal{Z}^{q}\left(Y^{\prime} ; T^{\prime}, *\right)^{\mathrm{ker}} \rightarrow \mathcal{Z}^{q}(Y ; T, *)^{\mathrm{ker}}$ to be defined, one requires that, for each $Y_{i} \in T, f\left(Y_{i}\right)$ must be contained in $Y_{j}^{\prime}$ for some $Y_{j}^{\prime} \in T^{\prime}$. Sending $\mathcal{Z}^{q}(Y ; T, *)_{\mathcal{S}}^{\mathrm{ker}}$ to the component $\mathcal{Z}^{q}(Y, *)_{\mathcal{S}}$ of $\mathcal{Z}^{q}(Y ; T, *)_{\mathcal{S}}$ by the inclusion gives the natural inclusion of complexes

$$
\mathcal{Z}^{q}(Y ; T, *)^{\mathrm{ker}} \rightarrow \mathcal{Z}^{q}(Y ; T, *)_{\mathcal{S}}
$$

\section{CyCle maps}

We describe the cycle class map from motivic cohomology to étale cohomology, as well as a relative version.

3.1. Functor complexes. Let $\mathrm{Sch}_{k}$ denote the category of $k$-schemes, essentially of finite type over $k$, and let $\mathcal{C}$ be a subcategory of $\mathrm{Sch}_{k}$. Suppose we have a functor $F: \mathcal{C}^{\mathrm{op}} \rightarrow \mathbf{C}(\mathbf{A b})$, where $\mathbf{C}(\mathbf{A b})$ is the additive category of cohomological complexes of abelian groups (with maps degree zero maps of complexes). We let $F_{N}$ denote the canonical truncation $\tau_{\leq N} F$.

Let $\Delta$ denote the category of order-preserving maps of the sets $[n]:=\{0<$ $\ldots<n\}, n=0,1, \ldots$ For a simplicial abelian group $A: \Delta^{\text {op }} \rightarrow \mathbf{A b}$, we view the associated chain complex as a cohomological complex $A^{*}$ in negative degrees, i.e., 
$A^{n}=A([-n])$, with differential the usual alternating sum. Similarly, for a simplicial object $F: \Delta^{\mathrm{op}} \rightarrow \mathbf{C}(\mathbf{A b})$, we have the double complex $F^{* *}$, with $F^{a, b}=F^{a}([-b])$.

For a cosimplicial scheme in $\mathcal{C}, X^{*}: \Delta \rightarrow \mathcal{C}$, we have the simplicial object $n \mapsto F\left(X^{n}\right)_{N}$ of $\mathbf{C}(\mathbf{A b})$; we let $F\left(X^{*}\right)_{N}$ denote the total complex of the associated double complex.

Let $F\left(X^{*}\right)$ be the inductive limit

$$
F\left(X^{*}\right):=\lim _{\vec{N}} F\left(X^{*}\right)_{N} .
$$

Sending $X^{*}$ to $F\left(X^{*}\right)$ or $F\left(X^{*}\right)_{N}$ defines a contravariant functor from the category of cosimplicial objects of $\mathcal{C}$ to $\mathbf{C}(\mathbf{A b})$, natural in $F$. We have convergent spectral sequences

$$
\begin{gathered}
E_{1}^{p, q}=H^{q}\left(F\left(X^{-p}\right)_{N}\right) \Longrightarrow H^{p+q}\left(F\left(X^{*}\right)_{N}\right) \\
E_{1}^{p, q}=H^{q}\left(F\left(X^{-p}\right)\right) \Longrightarrow H^{p+q}\left(F\left(X^{*}\right)\right) .
\end{gathered}
$$

In particular, if $\phi: F \rightarrow G$ is a natural transformation with $\phi(i)$ a quasi-isomorphism for each $i \in \mathcal{C}$ (for short, a quasi-isomorphism), then $\phi$ induces isomorphisms $H^{n}\left(F\left(X^{*}\right)_{N}\right) \rightarrow H^{n}\left(G\left(X^{*}\right)_{N}\right)$ and $H^{n}\left(F\left(X^{*}\right)\right) \rightarrow H^{n}\left(G\left(X^{*}\right)\right)$. Also, if $X^{*}$ is a constant cosimplicial scheme with value $X$, then the augmentation induces quasiisomorphisms $F(X)_{N} \rightarrow F\left(X^{*}\right)_{N}$ and $F(X) \rightarrow F\left(X^{*}\right)$.

Let $j: U^{*} \rightarrow X^{*}$ be an open cosimplicial subscheme in $\mathcal{C}$, and let $W^{*}$ denote the collection of closed complements $W^{n}=X^{n} \backslash U^{n}$; we call $W^{*}$ a closed subset of $X^{*}$. Suppose in addition we have closed cosimplicial subschemes $Y_{1}^{*}, \ldots, Y_{n}^{*}$ of $X^{*}$ (all relevant morphisms being in $\mathcal{C})$. Let $F\left(X^{*} ; Y_{1}^{*}, \ldots, Y_{n}^{*}\right)_{N}$ be the total complex of the double complex

$$
F\left(X^{*}\right)_{N} \rightarrow \bigoplus_{i=1}^{n} F\left(Y_{i}^{*}\right)_{N} \rightarrow \ldots \rightarrow \bigoplus_{|I|=j} F\left(Y_{I}^{*}\right)_{N} \rightarrow \ldots
$$

with $Y_{I}^{*}$ the cosimplicial closed subscheme $n \mapsto Y_{I}^{n}$, and the maps in the above complex defined as in $\S 2.4$. We set

$$
\begin{aligned}
& F_{W}\left(X^{*} ; Y_{1}^{*}, \ldots, Y_{n}^{*}\right)_{N} \\
& \quad:=\operatorname{cone}\left(j^{*}: F\left(X^{*} ; Y_{1}^{*}, \ldots, Y_{n}^{*}\right)_{N} \rightarrow F\left(U^{*} ; U^{*} \cap Y_{1}^{*}, \ldots, U^{*} \cap Y_{n}^{*}\right)_{N}\right)[-1] .
\end{aligned}
$$

Replacing $F_{N}$ with $F$ (or equivalently, taking the inductive limit over $N$ ) we have the complexes $F\left(X^{*} ; Y_{1}^{*}, \ldots, Y_{n}^{*}\right)$ and $F_{W}\left(X^{*} ; Y_{1}^{*}, \ldots, Y_{n}^{*}\right)$. As above, a quasiisomorphism $F \rightarrow G$ induces isomorphisms

$$
\begin{aligned}
H^{*}\left(F_{W}\left(X^{*} ; Y_{1}^{*}, \ldots, Y_{n}^{*}\right)_{N}\right) & \rightarrow H^{*}\left(G_{W}\left(X^{*} ; Y_{1}^{*}, \ldots, Y_{n}^{*}\right)_{N}\right) \\
H^{*}\left(F_{W}\left(X^{*} ; Y_{1}^{*}, \ldots, Y_{n}^{*}\right)\right) & \rightarrow H^{*}\left(G_{W}\left(X^{*} ; Y_{1}^{*}, \ldots, Y_{n}^{*}\right)\right) .
\end{aligned}
$$

Remark 3.2. A functor $F: \operatorname{Sch}_{k}^{\text {op }} \rightarrow \mathbf{C}(\mathbf{A b})$ is called homotopy invariant if the map

$$
F(X) \rightarrow F\left(X \times \mathbb{A}^{1}\right)
$$

induced by the projection is a quasi-isomorphism for all $X$. If $F$ is homotopy invariant, then, for $X^{*}=X \times \Delta^{*}$, the spectral sequences (3.1) and (3.1) have $E_{2}^{p, q}=0$ for $p<0$ and $E_{2}^{0, q}=H^{q}(F(X))$. One sees thereby that the augmentations $F(X)_{N} \rightarrow F\left(X \times \Delta^{*}\right)_{N}$ and $F(X) \rightarrow F\left(X \times \Delta^{*}\right)$ are quasi-isomorphisms. 
Examples 3.3. (1) For a $k$-scheme $X$, we let $G^{*}\left(X, \mu_{m}^{\otimes q}\right)$ denote the complex of abelian groups gotten by taking the global sections of the Godement resolution of the étale sheaf $\mu_{m}^{\otimes q}$ on $X$; for example, $G^{0}\left(\mu_{m}^{\otimes q}\right)_{X}$ is the product over all geometric points $x$ of $X$ of $H^{0}\left(k(x), \mu_{m}^{\otimes q}\right)$. Then sending $X$ to $G^{*}\left(X, \mu_{m}^{\otimes q}\right)$ defines the functor

$$
G^{*}\left(-, \mu_{m}^{\otimes q}\right): \operatorname{Sch}_{k}^{\mathrm{op}} \rightarrow \mathbf{C}^{+}(\mathbf{A b}) .
$$

The cohomology $H^{p}\left(G^{*}\left(X, \mu_{m}^{\otimes q}\right)\right)$ is canonically isomorphic to the étale cohomology $H_{\text {êt }}^{p}\left(X, \mu_{m}^{\otimes q}\right)$. The above discussion thus defines étale cohomology, with coefficients $\mu_{m}^{\otimes q}$, for a cosimplicial scheme $X^{*}$, as well as the relative version, with support in a closed subset.

(2) Let $X$ be a smooth quasi-projective $k$-scheme, $Y_{1}, \ldots, Y_{n}$ closed subschemes with $Y_{I}$ smooth for all $I$, and $\mathcal{S}$ a set of closed subsets of $X$, containing all the $Y_{I}$. Let $W$ be a closed subset of $X$, with complement $U$. Let $\mathcal{C}$ be the subcategory of $\operatorname{Sch}_{k}$ with objects the schemes $Y_{I} \times \Delta^{m}, m=0,1, \ldots$ The morphisms $Y_{I} \times \Delta^{m} \rightarrow Y_{J} \times \Delta^{l}$ are those of the form $i \times g$, with $i$ an inclusion, and $g$ the map induced by a map $[m] \rightarrow[l]$ in $\Delta$.

The construction described above gives us the functor $z_{\mathcal{S}}^{q}: \mathcal{C} \rightarrow \mathbf{A b}$ defined by

$$
z_{\mathcal{S}}^{q}\left(Y_{I} \times \Delta^{m}\right):=z^{q}\left(Y_{I}, m\right)_{\mathcal{S}} ;
$$

the complex $z_{\mathcal{S}, W}^{q}\left(X \times \Delta^{*} ; Y_{1} \times \Delta^{*}, \ldots, Y_{n} \times \Delta^{*}\right)[-2 q]$ is the relative cycle complex with support in $W, \mathcal{Z}_{W}^{q}\left(X ; Y_{1}, \ldots, Y_{n}, *\right)_{\mathcal{S}}$.

(3) For a normal crossing scheme $Y$, with irreducible components $Y_{1}, \ldots, Y_{n}$, let $\mathcal{S}$ be a set of closed subsets containing all the $Y_{I}$. Recall from $\S 2.5$ the cycle complex $\mathcal{Z}^{q}(Y, *)_{\mathcal{S}}$, and the group of cycles on $X$

$$
z^{q}(Y)_{\mathcal{S}}:=\mathcal{Z}^{q}(Y, 2 q)_{\mathcal{S}} .
$$

Explicitly, $z^{q}(Y)_{\mathcal{S}}$ is the kernel of the map

$$
\bigoplus_{i=1}^{n} z^{q}\left(Y_{i}\right)_{\mathcal{S}} \stackrel{\alpha}{\longrightarrow} \bigoplus_{1 \leq i<j \leq n} z^{q}\left(Y_{i, j}\right)_{\mathcal{S}}
$$

with $\alpha$ as in $\S 2.5$. If $W$ is a closed subset of $Y$, we let $z_{W}^{q}(Y)_{\mathcal{S}}$ be the subgroup of $z^{q}(Y)_{\mathcal{S}}$ consisting of cycles with support in $W$. We omit the $\mathcal{S}$ from the notation if $\mathcal{S}$ is the minimal choice, i.e., the set of all the $Y_{I}$.

If we take $\mathcal{C}$ to be the subcategory of $\mathrm{Sch}_{k}$ formed by the cosimplicial scheme $Y \times \Delta^{*}$, we form as in (2) the functor $z^{q}: \mathcal{C} \rightarrow \mathbf{A b}$, giving the identity

$$
z^{q}\left(Y \times \Delta^{*}\right)[-2 q]=\mathcal{Z}^{q}(Y, *) .
$$

Similarly, if $Z_{1}, \ldots, Z_{n}$ closed subschemes of $Y$ such that each $Z_{I}$ is a normal crossing scheme, let $\mathcal{C}$ be the category with the objects and morphisms defined as in (2) for the smooth case. We have the functor $z_{\mathcal{S}}^{q}: \mathcal{C} \rightarrow \mathbf{A b}$ as in (2), and the identity

$$
\mathcal{Z}^{q}\left(Y ; Z_{1}, \ldots, Z_{n}, *\right)=z_{\mathcal{S}}^{q}\left(Y \times \Delta^{*} ; Z_{1} \times \Delta^{*}, \ldots, Z_{n} \times \Delta^{*}\right)[-2 q] .
$$

3.4. Cohomology with support. Take $k$ to be a perfect field, let $X$ be a smooth $k$-variety, and $W$ a closed subset of $X$ of codimension $\geq q$. We denote the group of codimension $q$ cycles on $X$ with support in $W$ by $z_{W}^{q}(X)$. 
Remark 3.5. We require that $k$ be perfect so that a reduced closed subscheme $W$ of $X$ has a stratification by locally closed subschemes which are smooth over k, which enables us to use the standard purity results for étale cohomology. At the end of the day, we will remove this hypothesis by a limit argument.

Let $m$ be an integer prime to the characteristic of $k$. By the purity theorem for étale cohomology [14, Chap. VI, Theorem 5.1], there is a canonical isomorphism

$$
\operatorname{cyc}_{W}^{q}: z_{W}^{q}(X) \otimes \mathbb{Z} / m \rightarrow H_{W}^{2 q}\left(X, \mu_{m}^{\otimes q}\right),
$$

where $H_{W}^{2 q}$ is the étale cohomology with support in $W$. Indeed, if $W$ is smooth of codimension $q$, purity gives the canonical isomorphism

$$
H_{W}^{2 q}\left(X, \mu_{m}^{\otimes q}\right) \cong H^{0}(W, \mathbb{Z} / m) .
$$

The group $H^{0}(W, \mathbb{Z} / m)$ is just the free $\mathbb{Z} / m$-module on the irreducible components of $W$, which in turn is the $\mathbb{Z} / m$-module $z_{W}^{q}(X) \otimes \mathbb{Z} / m$. In addition, $H_{W}^{p}\left(X, \mu_{m}^{\otimes q}\right)=$ 0 for $p<2 q$. For arbitrary $W$ of codimenison $\geq q$, with singular locus $W_{\text {sing }}$, the localization sequence for étale cohomology with support [4] gives the isomorphism

$$
H_{W}^{2 q}\left(X, \mu_{m}^{\otimes q}\right) \cong H_{W \backslash W_{\text {sing }}}^{2 q}\left(X \backslash W_{\text {sing }}, \mu_{m}^{\otimes q}\right),
$$

which gives the isomorphism $\mathrm{cyc}_{W}^{q}$ in general.

The maps cyc $_{-}^{q}$ are natural with respect to pull-back by maps $f:(Y, T) \rightarrow$ $(X, W)$ such that $W \subset f(T), Y$ is smooth over $k$, and $T$ has codimension $\geq q$ on $Y$. The maps $\operatorname{cyc}_{W}^{q}$ are also natural with respect to proper push-forward, with the appropriate shift in the codimension, i.e., if $f:(Y, T) \rightarrow(X, W)$ is proper, of relative dimension $d$, with $f(T) \subset W$, and if $T$ has codimension $\geq q+d$ on $Y$, then

$$
\operatorname{cyc}_{W}^{q}\left(f_{*}(Z)\right)=f_{*}\left(\operatorname{cyc}_{T}^{q+d}(Z)\right)
$$

for $Z \in z_{T}^{q+d}(Y)$.

More generally, suppose that $Y$ is a normal crossing subscheme of a smooth $k$ scheme $X$, with irreducible components $T_{1}, \ldots, T_{r}$. Let $W$ be a closed subset of $Y$. For each index $J$, set

$$
W_{J}:=Y_{J} \cap W
$$

Lemma 3.6. Suppose that

$$
\operatorname{codim}_{Y_{J}}\left(W_{J}\right) \geq q
$$

for all indices $J$. Then

(1) $H_{W}^{p}\left(Y, \mu_{m}^{\otimes q}\right)=0$ for $p<2 q$.

(2) The sequence

$$
0 \rightarrow H_{W}^{2 q}\left(Y, \mu_{m}^{\otimes q}\right) \rightarrow \bigoplus_{j=1}^{m} H_{W_{j}, \text { ét }}^{2 q}\left(Y_{j}, \mu_{m}^{\otimes q}\right) \stackrel{\alpha}{\longrightarrow} \bigoplus_{1 \leq j<l \leq m}^{m} H_{W_{j, l}, \text { ét }}^{2 q}\left(Y_{j, l}, \mu_{m}^{\otimes q}\right)
$$

is exact, where $\alpha$ is defined as in $\S 2.4$

Proof. We have the Mayer-Vietoris spectral sequence [14, Chap. III, Exercise 2.24]

$$
E_{1}^{a, b}=\bigoplus_{|J|=a+1} H_{W_{J}}^{b}\left(Y_{J}, \mu_{m}^{\otimes q}\right) \Longrightarrow H_{W}^{a+b}\left(Y, \mu_{m}^{\otimes q}\right)
$$


By purity, we have $E_{1}^{a, b}=0$ for $b<2 q$; since $E_{1}^{a, b}=0$ for $a<0, E_{1}^{a, b}=0$ for $a+b<2 q$, whence (1). Similarly, the only non-zero terms with $a+b \leq 2 q+1$ and $b \leq 2 q$ are $E_{1}^{0,2 q}, E_{1}^{1,2 q}$. Thus

$$
E_{2}^{0,2 q}=E_{\infty}^{0,2 q}=H_{W}^{2 q}\left(Y, \mu_{m}^{\otimes q}\right)
$$

The map $\alpha$ is $d_{1}^{0,2 q}$, proving (2).

3.7. The cycle class map. We have the functor

$$
G^{*}\left(-, \mu_{m}^{\otimes q}\right): \operatorname{Sch}_{k} \rightarrow \mathbf{C}^{+}(\mathbf{A b})
$$

of Example 3.3, and its truncations

$$
G^{*}\left(-, \mu_{m}^{\otimes q}\right)_{N}:=\tau_{\leq N} G^{*}\left(-, \mu_{m}^{\otimes q}\right) \subset G^{*}\left(-, \mu_{m}^{\otimes q}\right)
$$

Let $X$ be a reduced $k$-scheme, and $\mathcal{S}$ a finite set of closed subsets of $X$, with $X \in \mathcal{S}$. Let $\mathcal{S}_{0}$ be a subset of $\mathcal{S}$ consisting of schemes equi-dimensional over $k$. Let $\mathcal{C}\left(\mathcal{S}_{0}, \mathcal{S}\right)$ be the subcategory of $\mathrm{Sch}_{k}$ with objects the schemes of the form $S \times \Delta^{m}$, with $S \in \mathcal{S}_{0}$. A morphism $S \times \Delta^{m} \rightarrow T \times \Delta^{l}$ is a map of the form $i \times g$, with $i: S \rightarrow T$ an inclusion, and with $g: \Delta^{m} \rightarrow \Delta^{l}$ the affine-linear map induced from some $\bar{g}:[m] \rightarrow[l]$ in $\Delta$.

Let $\left(S \times \Delta^{m}\right)_{\mathcal{S}}^{(q)}$ be the set of closed subsets $W$ of $S \times \Delta^{m}$ such that, for each $T \in \mathcal{S}, T \subset S$, and each face $F$ of $\Delta^{m}$, the intersection $W \cap(T \times F)$ has codimension $\geq q$ on $T \times F$. We let $G_{(q)}^{*}\left(S \times \Delta^{m}, \mu_{m}^{\otimes q}\right)_{\mathcal{S}}$ be the inductive limit

$$
\lim _{W \in\left(S \times \Delta^{m}\right)_{\mathcal{S}}^{(q)}} G_{W}^{*}\left(S \times \Delta^{m}, \mu_{m}^{\otimes q}\right) .
$$

Sending $S \times \Delta^{m}$ to $G_{(q)}^{*}\left(S \times \Delta^{m}, \mu_{m}^{\otimes q}\right)_{\mathcal{S}}$ defines the functor

$$
G_{(q)}^{*}\left(-, \mu_{m}^{\otimes q}\right): \mathcal{C}\left(\mathcal{S}_{0}, \mathcal{S}\right)^{\mathrm{op}} \rightarrow \mathbf{C}(\mathbf{A b})
$$

Assume that each $S \in \mathcal{S}_{0}$ is a normal crossing scheme. Let $z_{(q)}^{q}: \mathcal{C}\left(\mathcal{S}_{0}, \mathcal{S}\right)^{\text {op }} \rightarrow$ Ab be the functor

$$
z_{(q)}^{q}\left(S \times \Delta^{m}\right)=\lim _{W \in\left(S \times \Delta^{m}\right)_{\mathcal{S}}^{(q)}} z_{W}^{q}\left(S \times \Delta^{m}\right) ;
$$

the conditions on $S$ and $W$ imply that the relevant cycle intersection products are all defined, so that $z_{(q)}^{q}$ really is a functor.

We have the surjective natural transformation of functors

$$
G_{(q)}^{*}\left(-, \mu_{m}^{\otimes q}\right)_{N} \rightarrow H^{N}\left(G_{(q)}^{*}\left(-, \mu_{m}^{\otimes q}\right)\right)[-N] .
$$

Lemma 3.8. Suppose that

1. Each $S \in \mathcal{S}_{0}$ is a normal crossing scheme.

2. If $S \in \mathcal{S}_{0}$ has irreducible components $S_{1}, \ldots, S_{m}$, then each $S_{J}$ is in $\mathcal{S}_{0}$.

Then there is a unique isomorphism

$$
\operatorname{cyc}^{q}: z_{(q)}^{q} \otimes \mathbb{Z} / m \rightarrow H^{2 q}\left(G_{(q)}^{*}\left(-, \mu_{m}^{\otimes q}\right)\right)
$$


such that, for $S \in \mathcal{S}_{0}$ smooth, and $W \in\left(S \times \Delta^{m}\right)_{\mathcal{S}}^{(q)}$, the diagram

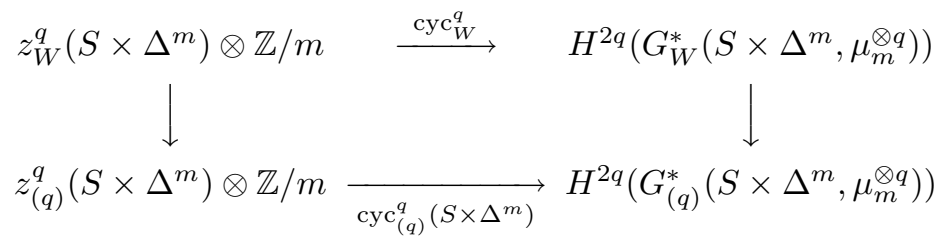

commutes. In addition, we have

$$
H^{p}\left(G_{(q)}^{*}\left(-, \mu_{m}^{\otimes q}\right)\right)=0
$$

for $p<2 q$.

Proof. Since $z_{(q)}^{q}\left(S \times \Delta^{m}\right)$ is the union of the $z_{W}^{q}\left(S \times \Delta^{m}\right)$, the uniqueness of $\operatorname{cyc}^{q}: z_{(q)}^{q} \otimes \mathbb{Z} / m$ is clear. The existence follows directly from Lemma 3.6, as does the cohomology vanishing for $p<2 q$.

We apply the lemma to construct a functorial cycle class map from motivic cohomology to étale cohomology. We first illustrate the simplest case, that of a smooth, quasi-projective variety $X$. We take $\mathcal{S}=\mathcal{S}_{0}=\{X\}$. We have the diagram of functors from $\mathcal{C}\left(\mathcal{S}_{0}, \mathcal{S}\right)^{\text {op }}$ to $\mathbf{C}(\mathbf{A b})$ :

$$
\begin{gathered}
z_{(q)}^{q}[-2 q] \otimes \mathbb{Z} / m \stackrel{\mathrm{cyc}^{q}}{\longrightarrow} H^{2 q}\left(G_{(q)}^{*}\left(-, \mu_{m}^{\otimes q}\right)\right)[-2 q] \\
\uparrow \alpha \\
G^{*}\left(-, \mu_{m}^{\otimes q}\right) \quad \stackrel{ }{\longleftarrow} \quad G_{(q)}^{*}\left(-, \mu_{m}^{\otimes q}\right)_{2 q},
\end{gathered}
$$

where $\alpha$ is the canonical map, and $\beta$ is the composition of the canonical inclusion $G_{(q)}^{*}\left(-, \mu_{m}^{\otimes q}\right)_{2 q} \rightarrow G_{(q)}^{*}\left(-, \mu_{m}^{\otimes q}\right)$, followed by the "forget the support" map $G_{(q)}^{*}\left(-, \mu_{m}^{\otimes q}\right) \rightarrow G^{*}\left(-, \mu_{m}^{\otimes q}\right)$. By Lemma 3.8, the map $\alpha$ is a quasi-isomorphism.

We now apply the above diagram of maps to the cosimplicial scheme $X \times \Delta^{*}$. By Example 3.3(2), we have

$$
z_{(q)}^{q}\left(X \times \Delta^{*}\right)[-2 q]=\mathcal{Z}^{q}(X, *) .
$$

By Remark 3.2, and the homotopy property for étale cohomology, the augmentation to $X$ induces a quasi-isomorphism

$$
G^{*}\left(X, \mu_{m}^{\otimes q}\right) \rightarrow G^{*}\left(X \times \Delta^{*}, \mu_{m}^{\otimes q}\right) .
$$

Taking cohomology, we have the mod $m$ cycle class map

$$
\mathrm{cl}^{q}: H^{*}(X, \mathbb{Z} / m(q)) \rightarrow H_{\text {et }}^{*}\left(X, \mu_{m}^{\otimes q}\right) .
$$

Assuming $X$ affine, we may enlarge the set $\mathcal{S}$ without changing the cohomology of $z_{(q)}^{q}\left(X \times \Delta^{*}\right)$; from this, one easily sees that $\mathrm{cl}^{q}$ is natural for affine $X$. We will discuss the naturality in general in the next section.

We may also modify the construction, by restricting the support throughout to a fixed closed subscheme $W$ of $X$. This gives the version with support in $W$,

$$
\mathrm{cl}^{q}: H_{W}^{*}(X, \mathbb{Z} / m(q)) \rightarrow H_{W}^{*}\left(X, \mu_{m}^{\otimes q}\right) .
$$

A similar construction gives an extension of the cycle class map to the setting of relative cohomology of normal crossing schemes (including the case of relative cohomology for smooth schemes). Indeed, let $Y$ be a normal crossing scheme, 
$Z_{1}, \ldots, Z_{n}$ closed normal crossing subschemes such that each $Z_{I}$ is a normal crossing scheme. Let $\mathcal{S}$ be a set of closed subsets of $Y$ containing all the $Z_{I}$, and let $\mathcal{S}_{0}$ be a subset of $\mathcal{S}$ satisfying the conditions of Lemma 3.8; one easily sees there always exists such an $\mathcal{S}_{0}$. We have the diagram (3.1) of functors from $\mathcal{C}(Y, \mathcal{S})^{\text {op }}$ to $\mathbf{C}(\mathbf{A b})$, with $\operatorname{cyc}^{q}[-2 q]$ an isomorphism, and $\alpha$ a quasi-isomorphism. We thus get a similar diagram upon evaluation at $\left(Y \times \Delta^{*} ; Z_{1} \times \Delta^{*}, \ldots, Z_{n} \times \Delta^{*}\right)$. The homotopy property for étale cohomology gives, as above, the quasi-isomorphism

$$
G^{*}\left(Y ; Z_{1}, \ldots, Z_{n}, \mu_{m}^{\otimes q}\right) \rightarrow G^{*}\left(Y \times \Delta^{*} ; Z_{1} \times \Delta^{*}, \ldots, Z_{n} \times \Delta^{*}, \mu_{m}^{\otimes q}\right) .
$$

Taking cohomology, we have the cycle class map

$$
\mathrm{cl}^{q}: H^{*}\left(Y ; Z_{1}, \ldots, Z_{n}, \mathbb{Z} / m(q)\right) \rightarrow H_{\text {êt }}^{*}\left(Y ; Z_{1}, \ldots, Z_{n}, \mu_{m}^{\otimes q}\right) .
$$

By construction, the cycle class map for relative cohomology is compatible with the maps in the long exact relativization sequences for motivic cohomology and étale cohomology. Similarly, the cycle class map is natural with respect to the restricted functoriality as described in $\S 2.5$. If $Y$ is smooth and affine, and if all the $Z_{I}$ are smooth, then the moving lemma for motivic cohomology [11, Chap. II, Theorem 3.5.14] allows us to increase $\mathcal{S}$, giving us naturality in general. Finally, since the cycle map with supports is covariantly functorial for proper maps, we have the commutative diagram

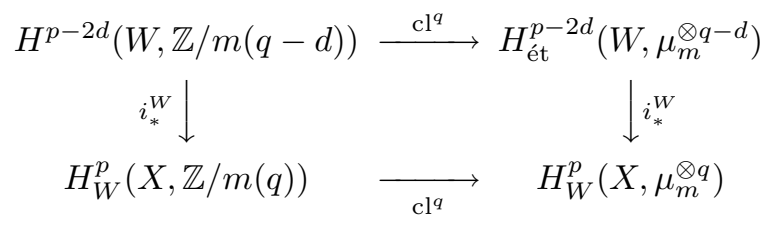

where $i_{*}^{W}: H^{p-2 d}(W, \mathbb{Z} / m(q-d)) \rightarrow H_{W}^{p}(X, \mathbb{Z} / m(q))$ is the Gysin isomorphism $(2.5)$, and $i_{*}^{W}: H_{\mathrm{ett}}^{p-2 d}\left(W, \mu_{m}^{\otimes q-d}\right) \rightarrow H_{W}^{p}\left(X, \mu_{m}^{\otimes q}\right)$ is the Gysin isomorphism in étale cohomology [14, Chap. IV, Corollary 6.4]. In consequence, the cycle class maps are compatible with the localization sequences for motivic cohomology and étale cohomology.

3.9. Motivic Complexes. We conclude the discussion of the cycle class map by giving a refinement of the cohomological cycle class map described above to a natural map in the derived category, $\mathbf{D}^{-}\left(\mathrm{Sh}_{X}\right)$ of Zariski sheaves on a smooth quasi-projective $k$-scheme $X$.

The complexes $\mathcal{Z}^{q}(-, *)$ are functorial for flat maps; in particular, for a smooth quasi-projective scheme $X$, we may form the complex of sheaves of abelian groups $U \mapsto \mathcal{Z}^{q}(U, *)$, which we denote by $\Gamma(q)_{X}$. The natural map

$$
\tau_{\leq q} \Gamma(q)_{X} \rightarrow \Gamma(q)_{X}
$$

is a quasi-isomorphism, since $H^{p}(R, \mathbb{Z}(q))=0$ if $p>q$, for a regular local $k$-agebra $R$.

Let $\epsilon: X_{\text {ét }} \rightarrow X_{\text {Zar }}$ be the change of topology map. Evaluating the diagram (3.1) on $U \times \Delta^{*}$ for $U$ open in $X$ and truncating gives the map in $\mathbf{D}^{-}\left(\mathrm{Sh}_{X}\right)$

$$
\mathrm{cl}_{X}^{q}: \Gamma(q)_{X} \otimes^{L} \mathbb{Z} / m \rightarrow \tau_{\leq q} R \epsilon_{*}\left(\mu_{m}^{\otimes q}\right),
$$

natural in $X$.

Since the complexes $\mathcal{Z}^{q}(U, *)$ satisfy Mayer-Vietoris for Zariski open covers, the natural map $H^{p}\left(\mathcal{Z}^{q}(X, *)\right) \rightarrow \mathbb{H}_{\text {Zar }}^{p}(X, \Gamma(q))$ is an isomorphism, and similarly with 
coefficients $\bmod m$. The cohomological cycle class map for $X$ is thus the map on $\mathbb{H}^{*}$ induced by $\mathrm{cl}_{X}^{q}$, followed by the natural map

$$
\mathbb{H}_{\text {Zar }}^{*}\left(X, \tau_{\leq q} R \epsilon_{*}\left(\mu_{m}^{\otimes q}\right)\right) \rightarrow \mathbb{H}_{\mathrm{Zar}}^{*}\left(X, R \epsilon_{*}\left(\mu_{m}^{\otimes q}\right)\right)=H_{\text {ét }}^{*}\left(X, \mu_{m}^{\otimes q}\right) .
$$

Remark 3.10. Since a smooth $k$-scheme is locally quasi-projective, the complex of sheaves $\Gamma(q)_{X}$ and the cycle map (3.3) have canonical extensions to essentially smooth $k$-schemes. All the properties of the cycle class map for smooth quasiprojective varieties described above are easily shown to extend to essentially smooth $k$-schemes.

\section{THE SEMI-LOCAL $n$-CUBE}

By taking relative motivic cohomology and relative étale cohomology on the semi-local $n$-cube, we relate the Bloch-Kato conjecture to the Beilinson-Lichtenbaum conjectures.

4.1. The semi-local $n$-cube. We write $\square_{n}$ for the affine space Spec $k\left[t_{1}, \ldots, t_{n}\right]$. Let $v$ be the set of points $\left(\epsilon_{1}, \ldots, \epsilon_{n}\right), \epsilon_{j} \in\{0,1\}$, of $\square_{n}, R_{n}$ the semi-local ring $\mathcal{O}_{\square_{n}, v}$ and $\hat{\square}_{n}$ the semi-local scheme $\operatorname{Spec} R_{n}$.

Let $\hat{\square}_{n}^{i ; \epsilon}$ be the subscheme of $\hat{\square}_{n}$ defined by the ideal $\left(t_{i}-\epsilon\right), \epsilon \in\{0,1\}$. We define the set of subschemes $T_{n}$ of $\hat{\square}_{n}$ by

$$
T_{n}:=\left\{\hat{\square}_{n}^{i ; \epsilon} \mid i=1, \ldots, n ; \epsilon \in\{0,1\}\right\} .
$$

We order the subschemes of $T_{n}$ by setting

$$
\hat{\square}_{n, s}:= \begin{cases}\hat{\square}_{n}^{s ; 1} & \text { for } s \leq n \\ \hat{\square}_{n}^{s-n ; 0} & \text { for } s>n,\end{cases}
$$

and let $T_{n}^{s}$ be the subset of $T_{n}$ consisting of the first $s$ subschemes. Let

$$
S_{n}:=T_{n}^{2 n-1}=T_{n}-\left\{\hat{\square}_{n}^{n ; 0}\right\} .
$$

We write $\partial^{s_{1}, \ldots, s_{r}} \hat{\square}_{n}$ for $\cup_{i=1}^{r} \hat{\square}_{n}^{s_{i}}$; we also write $\partial \hat{\square}_{n}$ for $\partial^{1, \ldots, 2 r} \hat{\square}_{n}$. As in $\S 2.4$, we write $\hat{\square}_{n, I}$ for the intersection $\cap_{s \in I}^{r} \hat{\square}_{n, s}$. Using the coordinates $t_{j}, j \notin I$, in the standard order identifies $\hat{\square}_{n, I}$ with $\hat{\square}_{n-|I|}$. Via this identification, we let $T_{n, I}^{s}$ denote the set of subschemes of $\hat{\square}_{n, I}$ corresponding to $T_{n-|I|}^{s}$.

4.2. Relativization sequences. Identifying $\hat{\square}_{n-1}$ with the face $\hat{\square}_{n, s}$ of $\hat{\square}_{n}$ gives us the fundamental relativization sequence

$$
\begin{aligned}
\rightarrow H^{p-1}\left(\hat{\square}_{n-1} ; T_{n-1}^{s-1}, \mathbb{Z} / m(q)\right) & \rightarrow H^{p}\left(\hat{\square}_{n} ; T_{n}^{s}, \mathbb{Z} / m(q)\right) \rightarrow \\
& H^{p}\left(\hat{\square}_{n} ; T_{n}^{s-1}, \mathbb{Z} / m(q)\right) \rightarrow H^{p}\left(\hat{\square}_{n-1} ; T_{n-1}^{s-1}, \mathbb{Z} / m(q)\right) \rightarrow
\end{aligned}
$$

We have similar sequences for étale cohomology. We will rely on the fundamental surjectivity property, proved in [7], Remark following Corollary 4.3,

Lemma 4.3. For all $n \geq 1$ and all $q \geq 0$, the restriction map

$$
H^{q}\left(\hat{\square}_{n} ; S_{n}, \mathbb{Z} / m(q)\right) \rightarrow H^{q}\left(\hat{\square}_{n-1} ; T_{n-1}, \mathbb{Z} / m(q)\right)
$$

is surjective. 
4.4. Cycle maps for $\hat{\square}_{n}$. We are now ready to relate the mod $n$ relative motivic cohomology and mod $n$ relative étale cohomology for $\hat{\square}_{n}$. We write 1 for the point $(1, \ldots, 1)$ of $\hat{\square}_{n}$.

We have the following version of a part of the Beilinson-Lichtenbaum conjectures [12]:

Conjecture $4.5\left(\mathrm{BL}_{a}\right)$. The cycle maps

$$
\mathrm{cl}^{a}: H^{p}(F, \mathbb{Z} / m(a)) \rightarrow H_{\text {ét }}^{p}\left(F, \mu_{m}^{\otimes a}\right)
$$

are isomorphisms for all $p \leq a$, and for all finitely generated field extensions $F$ of $k$.

Lemma 4.6. Suppose $\mathrm{BL}_{a}$ is true for $0 \leq a<q$. Then the cycle map

$$
\mathrm{cl}^{q}: H^{p}\left(\hat{\square}_{n} ; 1, \mathbb{Z} / m(q)\right) \rightarrow H_{\text {ét }}^{p}\left(\hat{\square}_{n} ; 1, \mu_{m}^{\otimes q}\right)
$$

is an isomorphism for all $p \leq q$.

Proof. Write $\mathbb{A}$ for $\mathbb{A}_{k}^{n}$, and let $\mathbb{A}^{(b)^{\prime}}$ denote the set of codimension points of $\mathbb{A}$ which are not in $\hat{\square}_{n}$. The standard constructions of Bloch-Ogus [4] give the augmented Gersten complex for motivic cohomology

$$
\begin{aligned}
0 \rightarrow H^{p}(\mathbb{A}, \mathbb{Z} / m(q)) \rightarrow H^{p}\left(\hat{\square}_{n}, \mathbb{Z} / m(q)\right) & \rightarrow \bigoplus_{x \in \mathbb{A}^{(1)^{\prime}}} H^{p-1}(k(x), \mathbb{Z} / m(q-1)) \\
& \rightarrow \ldots \rightarrow \bigoplus_{x \in \mathbb{A}^{(r)^{\prime}}} H^{p-r}(k(x), \mathbb{Z} / m(q-r)) \rightarrow \ldots
\end{aligned}
$$

and for étale cohomology

$$
\begin{aligned}
0 \rightarrow H_{\text {ét }}^{p}\left(\mathbb{A}, \mu_{m}^{\otimes q}\right) \rightarrow H_{\text {ét }}^{p}\left(\hat{\square}_{n}, \mu_{m}^{\otimes q}\right) \rightarrow & \bigoplus_{x \in \mathbb{A}^{(1)^{\prime}}} H_{\text {ét }}^{p-1}\left(k(x), \mu_{m}^{\otimes q-1}\right) \\
& \rightarrow \ldots \rightarrow \bigoplus_{x \in \mathbb{A}^{(r)^{\prime}}} H_{\text {ét }}^{p-r}\left(k(x), \mu_{m}^{\otimes q-r}\right) \rightarrow \ldots
\end{aligned}
$$

By the argument of [15], these complexes are exact.

Let $\pi: \mathbb{A} \rightarrow$ Spec $k$ be the structure morphism, and $i: 1 \rightarrow \hat{\square}_{n}$ the inclusion. The composition

$$
H_{\text {ét }}^{p}\left(k, \mu_{m}^{\otimes q}\right) \stackrel{\pi^{*}}{\longrightarrow} H_{\text {ét }}^{p}\left(\mathbb{A}, \mu_{m}^{\otimes q}\right) \rightarrow H_{\text {ét }}^{p}\left(\hat{\square}_{n}, \mu_{m}^{\otimes q}\right) \stackrel{i^{*}}{\longrightarrow} H_{\text {ét }}^{p}\left(k, \mu_{m}^{\otimes q}\right)
$$

being the identity, we may split off the first term in both the Gersten complexes, giving the exact Gersten complexes

$$
\begin{aligned}
0 \rightarrow H^{p}\left(\hat{\square}_{n} ; 1, \mathbb{Z} / m(q)\right) \rightarrow \bigoplus_{x \in \mathbb{A}^{(1)^{\prime}}} & H^{p-1}(k(x), \mathbb{Z} / m(q-1)) \rightarrow \ldots \\
& \rightarrow \bigoplus_{x \in \mathbb{A}^{(r)^{\prime}}} H^{p-r}(k(x), \mathbb{Z} / m(q-r)) \rightarrow \ldots
\end{aligned}
$$

and

$$
\begin{aligned}
& 0 \rightarrow H_{\text {êt }}^{p}\left(\hat{\square}_{n} ; 1, \mu_{m}^{\otimes q}\right) \rightarrow \bigoplus_{x \in \mathbb{A}^{(1)^{\prime}}} H_{\text {ét }}^{p-1}\left(k(x), \mu_{m}^{\otimes q-1}\right) \rightarrow \ldots \\
& \rightarrow \bigoplus_{x \in \mathbb{A}^{(r)^{\prime}}} H_{\text {ét }}^{p-r}\left(k(x), \mu_{m}^{\otimes q-r}\right) \rightarrow \ldots
\end{aligned}
$$


The compatibility of the cycle maps with localization implies that the various cycle maps give a map of complexes

$$
\mathrm{cl}^{*}:(4.1) \rightarrow(4.2) .
$$

This together with our hypothesis proves the lemma.

Lemma 4.7. Suppose that $\mathrm{BL}_{a}$ is true for $0 \leq a<q$. Then the cycle map

$$
\mathrm{cl}^{q}: H^{p}\left(\hat{\square}_{n} ; S_{n}, \mathbb{Z} / m(q)\right) \rightarrow H_{\text {ét }}^{p}\left(\hat{\square}_{n} ; S_{n}, \mu_{m}^{\otimes q}\right)
$$

is an isomorphism for all $p \leq q$.

Proof. We prove more generally that the cycle map

$$
\mathrm{cl}^{q}: H^{p}\left(\hat{\square}_{n} ; T_{n}^{s}, \mathbb{Z} / m(q)\right) \rightarrow H_{\text {ét }}^{p}\left(\hat{\square}_{n} ; T_{n}^{s}, \mu_{m}^{\otimes q}\right)
$$

is an isomorphism for all $p \leq q$ and for $1 \leq s<2 n$. We proceed by induction on $s$ and $n$.

We have the relativization sequence

$$
\rightarrow H^{p}\left(\hat{\square}_{n} ; T_{n}^{s}, \mathbb{Z} / m(q)\right) \rightarrow H^{p}\left(\hat{\square}_{n} ; T_{n}^{s-1}, \mathbb{Z} / m(q)\right) \rightarrow H^{p}\left(\hat{\square}_{n} ; T_{n-1}^{s-1}, \mathbb{Z} / m(q)\right) \rightarrow
$$

and a similar relativization sequence for étale cohomology. The appropriate cycle class maps define the map of the motivic sequence to the étale sequence; induction reduces us to the case $s=1$.

We have the relativization sequence

$$
\rightarrow H^{p}\left(\hat{\square}_{n} ; T_{n}^{1}, 1, \mathbb{Z} / m(q)\right) \rightarrow H^{p}\left(\hat{\square}_{n} ; T_{n}^{1}, \mathbb{Z} / m(q)\right) \rightarrow H^{p}(1 ; 1, \mathbb{Z} / m(q)) \rightarrow ;
$$

since $\mathcal{Z}^{q}(1 ; 1, *)$ is the cone on the identity map, we thus have the isomorphism

$$
H^{p}\left(\hat{\square}_{n} ; T_{n}^{1}, 1, \mathbb{Z} / m(q)\right) \cong H^{p}\left(\hat{\square}_{n} ; T_{n}^{1}, \mathbb{Z} / m(q)\right),
$$

and similarly for relative étale cohomology. The compatibility of the cycle class maps with the relativization sequence

$$
\rightarrow H^{p}\left(\hat{\square}_{n} ; T_{n}^{1}, 1, \mathbb{Z} / m(q)\right) \rightarrow H^{p}\left(\hat{\square}_{n} ; 1, \mathbb{Z} / m(q)\right) \rightarrow H^{p}\left(\hat{\square}_{n-1} ; 1, \mathbb{Z} / m(q)\right) \rightarrow,
$$

together with Lemma 4.6, shows that the cycle class map

$$
\mathrm{cl}^{q}: H^{p}\left(\hat{\square}_{n} ; T_{n}^{1}, 1, \mathbb{Z} / m(q)\right) \rightarrow H_{\text {ét }}^{p}\left(\hat{\square}_{n} ; T_{n}^{1}, 1, \mu_{m}^{\otimes q}\right)
$$

is an isomorphism in the desired range.

Lemma 4.8. Suppose that $\mathrm{BL}_{a}$ is true for $0 \leq a<q$. Suppose further that, for all finitely generated field extensions $F$ of $k$, the cycle maps

$$
\mathrm{cl}^{q}: H^{q}\left(\hat{\square}_{n} \times_{k} F ; T_{n} \times_{k} F, \mathbb{Z} / m(q)\right) \rightarrow H_{\text {ét }}^{q}\left(\hat{\square}_{n} \times_{k} F ; T_{n} \times_{k} F, \mu_{m}^{\otimes q}\right)
$$

are surjective for all $n$. Then

1. For all finitely generated field extensions $F$ of $k$, the cycle maps

$$
\mathrm{cl}^{q}: H^{p}\left(\hat{\square}_{n} \times_{k} F ; T_{n} \times_{k} F, \mathbb{Z} / m(q)\right) \rightarrow H_{\text {ét }}^{p}\left(\hat{\square}_{n} \times_{k} F ; T_{n} \times_{k} F, \mu_{m}^{\otimes q}\right)
$$

are isomorphisms for all $n \geq 1$ and all $p \leq q$.

2. $\mathrm{BL}_{q}$ is true. 
Proof. We first show how (1) implies (2). Since $m$ is prime to the characteristic of $k$, it suffices to prove (2) for the perfect closure of each finitely generated $F$; we may then replace $k$ with $F$, so it suffices to prove (2) for $F=k$. We have the relativization sequence

$$
\begin{aligned}
\rightarrow H^{p-1}(k, \mathbb{Z} / m(q)) \rightarrow H^{p}\left(\hat{\square}_{1} ; T_{1}, \mathbb{Z} / m(q)\right) & \rightarrow H^{p}\left(\hat{\square}_{1} ; S_{1}, \mathbb{Z} / m(q)\right) \\
& \rightarrow H^{p}(k, \mathbb{Z} / m(q)) \rightarrow
\end{aligned}
$$

and a similar sequence for the étale cohomology groups. The compatibility of the cycle class map with the relativitization sequences, together with (1) and Lemma 4.7 proves (2).

To prove (1), we have the relativization sequence

$$
H^{q}\left(\hat{\square}_{n+1} ; T_{n+1}, \mathbb{Z} / m(q)\right) \rightarrow H^{q}\left(\hat{\square}_{n+1} ; S_{n+1}, \mathbb{Z} / m(q)\right) \rightarrow H^{q}\left(\hat{\square}_{n} ; T_{n}, \mathbb{Z} / m(q)\right) \rightarrow 0,
$$

where the surjectivity is given by Lemma 4.3. We have a similar sequence for étale cohomology, possibly without the surjectivity on the right. The map

$$
\mathrm{cl}^{q}: H^{q}\left(\hat{\square}_{n} ; S_{n}, \mathbb{Z} / m(q)\right) \rightarrow H_{\text {ét }}^{q}\left(\hat{\square}_{n} ; S_{n}, \mu_{m}^{\otimes q}\right)
$$

is an isomorphism by Lemma 4.7 , hence surjectivity of

$$
\mathrm{cl}^{q}: H^{q}\left(\hat{\square}_{n+1} ; T_{n+1}, \mathbb{Z} / m(q)\right) \rightarrow H_{\text {ét }}^{q}\left(\hat{\square}_{n+1} ; T_{n+1}, \mu_{m}^{\otimes q}\right)
$$

implies injectivity of

$$
\mathrm{cl}^{q}: H^{q}\left(\hat{\square}_{n} ; T_{n}, \mathbb{Z} / m(q)\right) \rightarrow H_{\text {ét }}^{q}\left(\hat{\square}_{n} ; T_{n}, \mu_{m}^{\otimes q}\right) .
$$

We now proceed by descending induction on the cohomology degree. Fix an integer $p<q$, and suppose that $\mathrm{cl}^{q}: H^{a}\left(\hat{\square}_{n} ; T_{n}, \mathbb{Z} / m(q)\right) \rightarrow H_{\text {êt }}^{a}\left(\hat{\square}_{n} ; T_{n}, \mu_{m}^{\otimes q}\right)$ is an isomorphism for all $a$ with $p+1 \leq a \leq q$ and for all $n$. We have the relativization sequence

$$
\begin{aligned}
& H^{p}\left(\hat{\square}_{n+1} ; T_{n+1}, \mathbb{Z} / m(q)\right) \rightarrow H^{p}\left(\hat{\square}_{n+1} ; S_{n+1}, \mathbb{Z} / m(q)\right) \\
& \rightarrow H^{p}\left(\hat{\square}_{n} ; T_{n}, \mathbb{Z} / m(q)\right) \rightarrow H^{p+1}\left(\hat{\square}_{n+1} ; T_{n+1}, \mathbb{Z} / m(q)\right) \\
& \quad \rightarrow H^{p+1}\left(\hat{\square}_{n+1} ; S_{n+1}, \mathbb{Z} / m(q)\right) \rightarrow
\end{aligned}
$$

and also for étale cohomology. As we have the isomorphism

$$
\mathrm{cl}^{q}: H^{a}\left(\hat{\square}_{n+1} ; S_{n+1}, \mathbb{Z} / m(q)\right) \rightarrow H_{\text {ét }}^{a}\left(\hat{\square}_{n+1} ; S_{n+1}, \mu_{m}^{\otimes q}\right)
$$

for all $a \leq q$, the map $\mathrm{cl}^{q}: H^{p}\left(\hat{\square}_{n} ; T_{n}, \mathbb{Z} / m(q)\right) \rightarrow H_{\text {ét }}^{p}\left(\hat{\square}_{n} ; T_{n}, \mu_{m}^{\otimes q}\right)$ is surjective; as $n$ is arbitrary, the map

$$
\operatorname{cl}^{q}: H^{p}\left(\hat{\square}_{n+1} ; T_{n+1}, \mathbb{Z} / m(q)\right) \rightarrow H_{\text {ét }}^{p}\left(\hat{\square}_{n+1} ; T_{n+1}, \mu_{m}^{\otimes q}\right)
$$

is surjective as well, hence $\mathrm{cl}^{q}: H^{p}\left(\hat{\square}_{n} ; T_{n}, \mathbb{Z} / m(q)\right) \rightarrow H_{\text {êt }}^{p}\left(\hat{\square}_{n} ; T_{n}, \mu_{m}^{\otimes q}\right)$ is injective.

\section{SurJeCtivity}

We now complete the discussion, by showing how the Bloch-Kato conjecture for fields implies the surjectivity condition of Lemma 4.8. 
5.1. Let $\mathcal{C}$ be a subcategory of $\mathrm{Sch}_{k}$, and $F: \mathcal{C}^{\mathrm{op}} \rightarrow \mathbf{C}(\mathbf{A b})$ a functor. As in $\S 3.1$, if we have a $k$-scheme $X$ with closed subschemes $Y_{1}, \ldots, Y_{m}$, such that all the inclusions $Y_{I} \rightarrow Y_{J}, J \subset I \subset\{1, \ldots, n\}$ are in $\mathcal{C}$, we form the relative complex $F\left(X ; Y_{1}, \ldots, Y_{n}\right)$, which is defined as the total complex of the double complex

$$
F(X) \rightarrow \bigoplus_{i=1}^{n} F\left(Y_{i}\right) \rightarrow \ldots \rightarrow \bigoplus_{|I|=j} F\left(\left(Y_{I}\right) \rightarrow \ldots\right.
$$

with $F^{i}\left(Y_{I}\right)$ in total degree $i+|I|$. The relative complexes $F\left(X ; Y_{1}, \ldots, Y_{n}\right)$ are natural in $F$, and have the functorialities described in $§ 2.5$. We have as well the subcomplex

$$
F\left(X ; Y_{1}, \ldots, Y_{n}\right)^{\mathrm{ker}}:=\operatorname{ker}\left(F(X) \rightarrow \bigoplus_{i=1}^{n} F\left(Y_{i}\right)\right)
$$

of $F\left(X ; Y_{1}, \ldots, Y_{n}\right)$.

5.2. The category $\mathcal{C}(n)$. For $1 \leq s \leq n$ let

$$
\begin{aligned}
& i_{s}:\left(t_{1}, \ldots, t_{n-1}\right) \mapsto\left(t_{1}, \ldots, t_{s-1}, 0, t_{s}, \ldots, t_{n-1}\right) \\
& j_{s}:\left(t_{1}, \ldots, t_{n-1}\right) \mapsto\left(t_{1}, \ldots, t_{s-1}, 1, t_{s}, \ldots, t_{n-1}\right)
\end{aligned}
$$

be the inclusion of the faces $t_{s}=0$ and $t_{s}=1$ into $\hat{\square}_{n}$. We have the identities

$$
i_{t} i_{s}=\left\{\begin{array}{ll}
i_{s+1} i_{t} & s \geq t \\
i_{s} i_{t-1} & s<t
\end{array} ; \quad j_{t} i_{s}=\left\{\begin{array}{ll}
i_{s+1} j_{t} & s \geq t \\
i_{s} j_{t-1} & s<t
\end{array} ; \quad j_{t} j_{s}=\left\{\begin{array}{ll}
j_{s+1} j_{t} & s \geq t \\
j_{s} j_{t-1} & s<t
\end{array} .\right.\right.\right.
$$

Similarly, we define projection maps for $1 \leq s \leq n$ and $1 \leq s<n$, respectively,

$$
\begin{aligned}
p_{s}:\left(t_{1}, \ldots, t_{n}\right) & \mapsto\left(t_{1}, \ldots, t_{s-1}, t_{s+1}, \ldots, t_{n}\right) \\
q_{s}:\left(t_{1}, \ldots, t_{n}\right) & \mapsto\left(t_{1}, \ldots, t_{s-1}, 1-\left(t_{s}-1\right)\left(t_{s+1}-1\right), t_{s+2}, \ldots, t_{n}\right) .
\end{aligned}
$$

The following identities hold

$$
\begin{gathered}
p_{s} j_{t}=\left\{\begin{array}{ll}
j_{t-1} p_{s} & t>s \\
\mathrm{id} & t=s \\
j_{t} p_{s-1} & t<s
\end{array} ; \quad p_{s} i_{t}= \begin{cases}i_{t-1} p_{s} & t>s \\
\mathrm{id} & t=s \\
i_{t} p_{s-1} & t<s\end{cases} \right. \\
q_{s} j_{t}=\left\{\begin{array}{ll}
j_{t-1} q_{s} & t>s+1 \\
j_{s} p_{s} & t=s, s+1 ; \\
j_{t} q_{s-1} & t<s
\end{array} ; \quad q_{s} i_{t}= \begin{cases}i_{t-1} q_{s} & t>s+1 \\
\mathrm{id} & t=s, s+1 . \\
i_{t} q_{s-1} & t<s\end{cases} \right.
\end{gathered}
$$

We let $\mathcal{C}(n)$ be the subcategory of $\operatorname{Sch}_{k}$ with objects the subschemes of $\hat{\square}_{n}$ of the form $\partial^{s_{1}, \ldots, s_{r}} \hat{\square}_{n}$. A morphism $f: \partial^{s_{1}, \ldots, s_{r}} \hat{\square}_{n} \rightarrow \partial^{s_{1}^{\prime}, \ldots, s_{r^{\prime}}^{\prime}} \hat{\square}_{n}$ is a map of $k$-schemes such that, for each face $\partial^{s_{1}, \ldots, s_{r}} \hat{\square}_{n, I}$ of $\partial^{s_{1}, \ldots, s_{r}} \hat{\square}_{n}$, there is a face $\partial^{s_{1}^{\prime}, \ldots, s_{r^{\prime}}^{\prime}} \hat{\square}_{n, I^{\prime}}$ of $\partial^{s_{1}^{\prime}, \ldots, s_{r^{\prime}}^{\prime}} \hat{\square}_{n}$ such that the restriction of $f$ to $\partial^{s_{1}, \ldots, s_{r}} \hat{\square}_{n, I}$ factors as

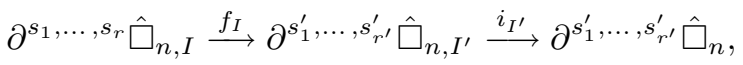

with $f_{I}$ flat, and $i_{I^{\prime}}$ the inclusion. 
Proposition 5.3. For all $s \leq 2 n$, the canonical maps

$$
F\left(\hat{\square}_{n}, T_{n}^{s}\right)^{\mathrm{ker}} \rightarrow F\left(\hat{\square}_{n}, T_{n}^{s}\right) ; \quad F\left(\partial \hat{\square}_{n}, T_{n}^{s}\right)^{\mathrm{ker}} \rightarrow F\left(\partial \hat{\square}_{n}, T_{n}^{s}\right)
$$

are quasi-isomorphisms. For all $s<2 n$, the inclusions

$$
F\left(\hat{\square}_{n}, T_{n}^{s}\right)^{\mathrm{ker}} \subseteq F\left(\hat{\square}_{n}, T_{n}^{s-1}\right)^{\mathrm{ker}} ; \quad F\left(\partial \hat{\square}_{n}, T_{n}^{s}\right)^{\mathrm{ker}} \subseteq F\left(\partial \hat{\square}_{n}, T_{n}^{s-1}\right)^{\mathrm{ker}}
$$

are split; the splittings are natural in $F$.

Proof. We can assume that the proposition holds for $\hat{\square}_{n-1}$ and proceed by induction on $s$. We will prove the proposition for $\partial \hat{\square}_{n}$; the proof for $\hat{\square}_{n}$ is exactly the same, replacing $\partial \hat{\square}_{n}$ with $\hat{\square}_{n}$, and is left to the reader.

By contravariant functoriality, there are maps

$$
\begin{aligned}
i_{s}^{*}, j_{s}^{*}: F\left(\partial \hat{\square}_{n}\right) \rightarrow F\left(\hat{\square}_{n-1, s}\right) \\
p_{s}^{*}, q_{s}^{*}: F\left(\hat{\square}_{n-1, s}\right) \rightarrow F\left(\partial \hat{\square}_{n}\right) .
\end{aligned}
$$

For $s \leq 2 n$, consider the following commutative diagram of complexes:

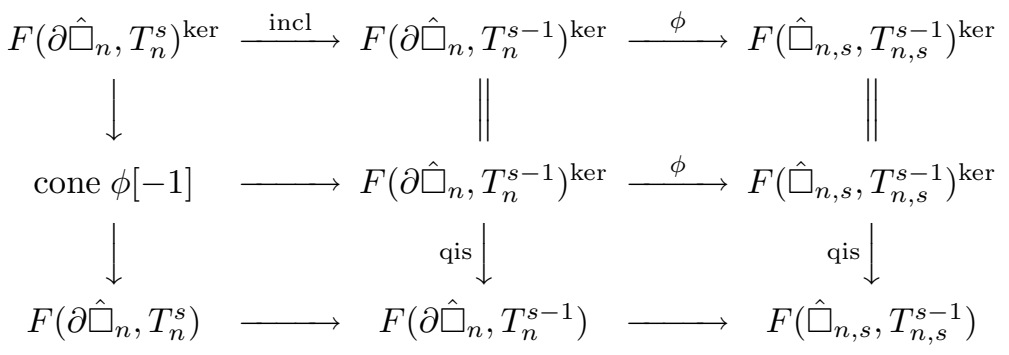

If we can show that the map $\phi$ is naturally split surjective, then the second statement of the proposition follows, and all rows of the diagram are distinguished triangles of complexes (in fact the upper row is a short exact sequence of complexes). Since by induction the two right vertical maps are quasi-isomorphisms, the left vertical maps will be a quasi-isomorphism as well.

Consider the following diagram, where $\iota_{s}=j_{s}^{*}, i_{s-n}^{*}$ for $s \leq n$ and $s>n$, respectively, and $\rho_{s}=p_{s}^{*}, q_{s-n}^{*}$ for $s \leq n$ and $s>n$, respectively.

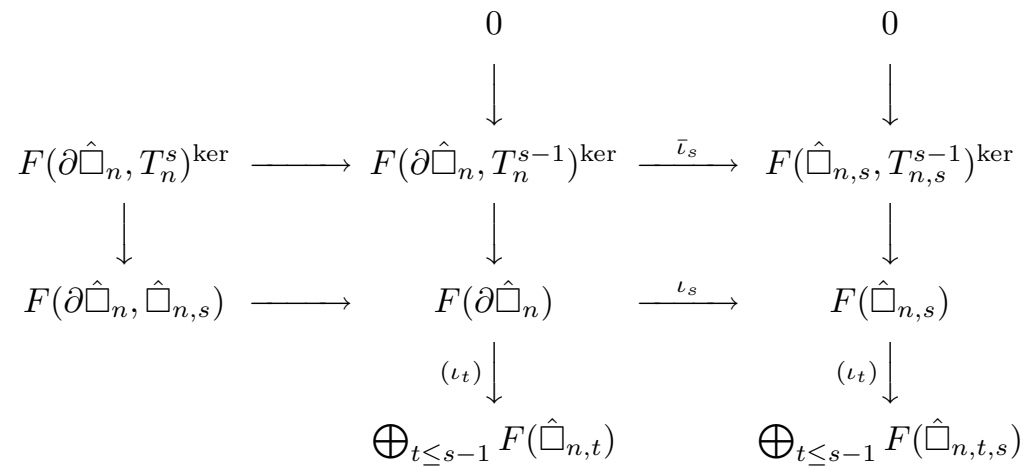

Let $\alpha$ be an element of $F\left(\hat{\square}_{n, s}, T_{n, s}^{s-1}\right)$ ker. Then $\alpha$ can be viewed as an element of $F\left(\hat{\square}_{n, s}\right)$ mapping to zero under $\iota_{t}$ for $t<s$. Lift $\alpha$ to $\bar{\alpha}$ in $F\left(\partial \hat{\square}_{n}\right)$ by $\rho_{s}$. We have to show that $\bar{\alpha}$ lies in the kernel of $\iota_{t}$ for $t<s$. But this follows from (5.2) for $t<s \leq n$ :

$$
\iota_{t} \bar{\alpha}=j_{t}^{*} p_{s}^{*} \alpha=p_{s-1}^{*} j_{t}^{*} \alpha=0
$$


For $s>t>n$ this follows from (5.3):

$$
\iota_{t} \bar{\alpha}=i_{t-n}^{*} q_{s-n}^{*} \alpha=q_{s-n-1}^{*} i_{t-n}^{*} \alpha=0
$$

and for $t \leq n<s$,

$$
\iota_{t} \bar{\alpha}=j_{t}^{*} q_{s-n}^{*} \alpha= \begin{cases}q_{s}^{*} j_{t-1}^{*} \alpha=0 & t \geq s-n+1 \\ p_{s-n}^{*} j_{t}^{*} \alpha=0 & t=s-n, s-n+1 \\ q_{s-n-1}^{*} j_{t}^{*} \alpha=0 & t<s-n .\end{cases}
$$

Proposition 5.4. Let $X=\operatorname{Spec} R$ be a semi-local $k$-scheme, where $R$ is a localization of a k-algebra of finite type. Then each element $\eta$ of $H_{\text {ét }}^{p}\left(X, \mu_{m}^{\otimes q}\right)$ is of the form $i^{*} \tau$, where $i: X \rightarrow Y$ is a morphism of $X$ to a smooth finite type semi-local $k$-scheme, and $\tau$ is in $H_{\text {êt }}^{q}\left(Y, \mu_{m}^{\otimes q}\right)$.

Proof. Take a closed embedding of $X$ into the semi-localization $A$ of an affine space $\mathbb{A}_{k}^{N}$, and let $X_{h}$ be the henselization of $A$ along $X$. Let $i_{h}: X \rightarrow X_{h}$ be the inclusion and let $\mathcal{F}$ be a torsion étale sheaf on $X_{h}$. It follows from [6, Theorem 1] (see also [16]) that

$$
i_{h}^{*}: H_{\text {ét }}^{p}\left(X_{h}, \mathcal{F}\right) \rightarrow H_{\text {ét }}^{p}\left(X, i_{h}^{*} \mathcal{F}\right)
$$

is an isomorphism. Since $X$ and $A$ are affine, $X_{h}$ is a filtered projective limit of smooth semi-local $k$-schemes of finite type. Since $H_{\text {ét }}^{p}\left(-, \mu_{m}^{\otimes q}\right)$ maps filtered projective limits to filtered inductive limits, the result follows.

Lemma 5.5. Suppose that map $\mathrm{cl}^{q}: H^{q}(F, \mathbb{Z} / m(q)) \rightarrow H_{\mathrm{ett}}^{q}\left(F, \mu_{m}^{\otimes q}\right)$ is a surjection for all fields $F$ finitely generated over $k$, and that the map $\mathrm{cl}^{q-1}: H^{q-1}(F, \mathbb{Z} / m(q-$ $1)) \rightarrow H_{\text {ét }}^{q-1}\left(F, \mu_{m}^{\otimes q-1}\right)$ is injective for all fields $F$ finitely generated over $k$. Then the cycle class

$$
\mathrm{cl}^{q}: H^{q}\left(\hat{\square}_{n} ; T_{n}, \mathbb{Z} / m(q)\right) \rightarrow H_{\mathrm{ét}}^{q}\left(\hat{\square}_{n} ; T_{n}, \mu_{m}^{\otimes q}\right)
$$

is surjective for all $n$.

Proof. Let $R$ be the semi-local ring of finitely many smooth points on a algebraic variety $X$. By Bloch-Ogus theory [4], we have the exact Gersten complex

$$
\begin{aligned}
0 \rightarrow H^{q}(R, \mathbb{Z} / m(q)) & \rightarrow H^{q}(k(X), \mathbb{Z} / m(q)) \rightarrow \ldots \\
& \rightarrow \bigoplus_{x \in(\operatorname{Spec} R)^{(a)}} H^{q-a}(k(x), \mathbb{Z} / m(q-a)) \rightarrow \ldots
\end{aligned}
$$

and a similar exact Gersten complex for étale cohomology. From the compatibility of the cycle class maps, and our assumption, the cycle class map $\mathrm{cl}^{q}$ : $H^{q}(R, \mathbb{Z} / m(q)) \rightarrow H_{\mathrm{ét}}^{q}\left(R, \mu_{m}^{\otimes q}\right)$ is surjective. By Lemma 5.4, and the functoriality of the cycle class map, the map cl ${ }^{q}: H^{q}\left(\partial \hat{\square}_{n+1}, \mathbb{Z} / m(q)\right) \rightarrow H_{\text {ét }}^{q}\left(\partial \hat{\square}_{n+1}, \mu_{m}^{\otimes q}\right)$ 
is surjective for all $n$. We have the commutative diagram

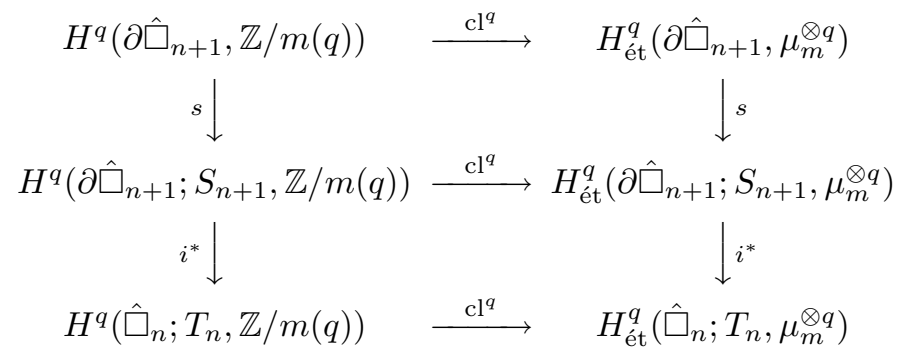

where $s$ is the natural splitting given by Lemma 5.3, and $i$ is the inclusion of the face $t_{n+1}=0$.

We recall that étale cohomology satisfies Mayer-Vietoris for unions of closed subschemes. Indeed, suppose $Z=Z_{1} \cup Z_{2}$, with $Z_{i}$ closed in $Z$, let $p: Z_{1} \amalg Z_{2} \rightarrow Z$ be the evident map, and let $i_{*}: Z_{1} \cap Z_{2} \rightarrow Z$ be the inclusion. Since $p$ is finite, we have

$$
H^{*}\left(Z, p_{*}\left(\mu_{m}^{\otimes q}\right)\right)=H^{*}\left(Z_{1}, \mu_{m}^{\otimes q}\right) \oplus H^{*}\left(Z_{2}, \mu_{m}^{\otimes q}\right) ;
$$

similarly, we have

$$
H^{*}\left(Z, i_{*}\left(\mu_{m}^{\otimes q}\right)\right)=H^{*}\left(Z_{1} \cap Z_{2}, \mu_{m}^{\otimes q}\right) .
$$

The exact sheaf sequence

$$
0 \rightarrow \mu_{m}^{\otimes q} \rightarrow p_{*}\left(\mu_{m}^{\otimes q}\right) \rightarrow i_{Z_{1} \cap Z_{2} *} \mu_{m}^{\otimes q} \rightarrow 0
$$

thus gives the desired Mayer-Vietoris sequence.

The Mayer-Vietoris property readily implies that the map

$$
i^{*}: H_{\text {êt }}^{q}\left(\partial \hat{\square}_{n+1} ; S_{n+1}, \mu_{m}^{\otimes q}\right) \rightarrow H_{\text {êt }}^{q}\left(\hat{\square}_{n} ; T_{n}, \mu_{m}^{\otimes q}\right)
$$

is an isomorphism, whence the lemma.

5.6. The main theorem. We can now give the proof of our main result Theorem 1.1. We proceed by induction on $q$, the case $q=0$ being trivially satisfied.

Lemma 5.7. The surjectivity of

$$
\operatorname{cl}_{F}^{q}: H^{q}(F, \mathbb{Z} / m(q)) \rightarrow H_{\text {ét }}^{q}\left(F, \mu_{m}^{\otimes q}\right)
$$

for all fields $F$ finitely generated over $k$ implies the surjectivity of

$$
\mathrm{cl}_{F}^{a}: H^{a}(F, \mathbb{Z} / m(a)) \rightarrow H_{\text {ét }}^{a}\left(F, \mu_{m}^{\otimes a}\right)
$$

for all fields $F$ finitely generated over $k$ and for all $a$ with $0 \leq a \leq q$.

Proof. Let $R$ be the local ring $F[X]_{(X)}$, let $\eta$ be in $H_{\text {ét }}^{a}\left(F, \mu_{m}^{\otimes a}\right)$, and assume that $\mathrm{cl}_{F(X)}^{a+1}$ is surjective. Let $p^{*}: H_{\text {ét }}^{a}\left(F, \mu_{m}^{\otimes a}\right) \rightarrow H_{\text {ét }}^{a}\left(F(X), \mu_{m}^{\otimes a}\right)$ be the map induced by the inclusion $F \rightarrow F(X)$, and let $\omega=\operatorname{cl}^{1}(X) \cup p^{*} \eta$. We have the commutative diagram

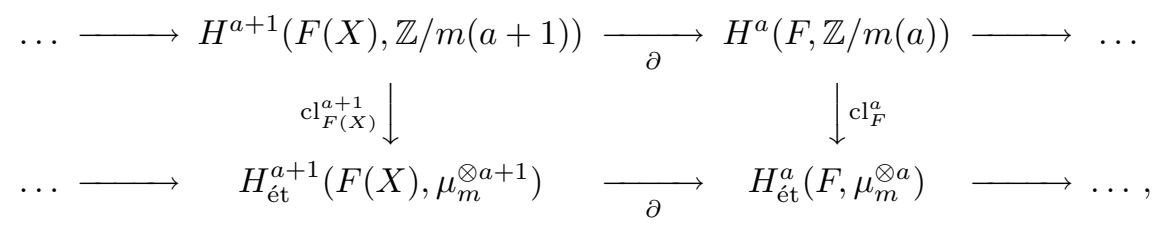


where the rows are the boundary maps in the localization sequence for the open complement $\operatorname{Spec} F(X)$ of $\operatorname{Spec} F$ in $\operatorname{Spec} F[X]_{(X)}$. For $a=0$, we have canonical isomorphisms

$$
H^{0}(F, \mathbb{Z} / m(0)) \cong \mathbb{Z} / m \cong H_{\text {ét }}^{a}(F, \mathbb{Z} / m),
$$

and $\mathrm{cl}_{F}^{0}$ is the identity. In addition, the map

$$
\partial: H^{1}(F(X), \mathbb{Z}(1)) \cong F(X)^{\times} \rightarrow H^{0}(F, \mathbb{Z})
$$

is just the classical divisor map, hence

$$
\partial\left(\operatorname{cl}^{1}(X)\right)=\operatorname{cl}^{0}(\partial(X))=\operatorname{cl}^{0}(1)=1 .
$$

Since the boundary map is a $H_{\mathrm{ett}}^{*}\left(F, \mu_{m}^{\otimes *}\right)$-module homomorphism, this gives the identity $\eta=\partial(\omega)$. By assumption, there is an element $z \in H^{a+1}(F(X), \mathbb{Z} / m(a+1))$ with $\mathrm{cl}^{a+1}(z)=\omega$, giving

$$
\mathrm{cl}_{F}^{a}(\partial z)=\partial\left(\mathrm{cl}_{F(X)}^{a+1}(z)\right)=\partial(\omega)=\eta .
$$

Applying our induction hypothesis, it follows from Lemma 5.7 that the map

$$
\mathrm{cl}_{X}^{a}: H^{p}(X, \mathbb{Z} / m(a)) \rightarrow H_{\text {ét }}^{p}\left(X, \mu_{m}^{\otimes a}\right)
$$

is an isomorphism for all $X$ smooth and quasi-projective over $k$, and for $p \leq a<$ $q$. Since both $H^{p}(X, \mathbb{Z} / m(a))$ and $H_{\text {ét }}^{p}\left(X, \mu_{m}^{\otimes a}\right)$ take filtered projective limits of schemes (with flat transition maps) to filtered inductive limits, it follows that

$$
\mathrm{cl}_{F}^{a}: H^{p}(F, \mathbb{Z} / m(a)) \rightarrow H_{\text {ét }}^{p}\left(F, \mu_{m}^{\otimes a}\right)
$$

is an isomorphism for all fields $F$ finitely generated over $k$, and for $p \leq a<q$ (we are indebted to B. Kahn for pointing out this reduction).

Let $R$ be the local ring of a smooth point on a $k$-variety $X$ of finite type. To prove the main theorem, it suffices to show that the map

$$
\mathrm{cl}^{q}: H^{p}(R, \mathbb{Z} / m(q)) \rightarrow H_{\text {ét }}^{p}\left(R, \mu_{m}^{\otimes q}\right)
$$

is an isomorphism for all $p \leq q$.

By comparing the Gersten resolutions

$$
\begin{aligned}
0 \rightarrow H^{p}(R, \mathbb{Z} / m(q)) \rightarrow H^{p}(k(X), \mathbb{Z} / & m(q)) \\
& \rightarrow \bigoplus_{x \in \operatorname{Spec} R^{(a)}} H^{p-a}(R, \mathbb{Z} / m(q-a)) \rightarrow \ldots \\
0 \rightarrow H_{\text {ét }}^{p}\left(R, \mu_{m}^{\otimes q}\right) \rightarrow H_{\text {ét }}^{p}\left(k(X), \mu_{m}^{\otimes q}\right) & \rightarrow \ldots \\
& \rightarrow \bigoplus_{x \in \operatorname{Spec} R^{(a)}} H_{\text {ét }}^{p-a}\left(R, \mu_{m}^{\otimes q-a}\right) \rightarrow \ldots
\end{aligned}
$$

via the maps $\mathrm{cl}^{*}$ and using the isomorphisms (5.6), we reduce to the case of a finitely generated field $F$ over $k$. Lemma 4.8 and Lemma 5.5 together with the isomorphisms (5.6) complete the proof.

Remark 5.8. The idea of using Gabber's rigidity theorem to pass from a version of the Bloch-Kato conjecture for singular schemes to the usual version is due to $\mathrm{R}$. Hoobler, who used this argument to extend the Merkerjev-Suslin theorem (which is the Bloch-Kato conjecture for weight two) to arbitrary local rings. See [5]. 
Remark 5.9. Let $\Gamma_{X}(q)$ ét denote the sheafification of $\Gamma_{X}(q)$ for the étale topology on $X$. One can rephrase the conclusion of Theorem 1.1 as the assertion that the natural map

$$
\Gamma_{X}(a) \otimes^{L} \mathbb{Z} / m \rightarrow \tau_{\leq a} R \epsilon_{*} \Gamma_{X}(a)_{\text {ét }} \otimes^{L} \mathbb{Z} / m
$$

is a quasi-isomorphism for $0 \leq a \leq q$. Indeed, it follows from the rigidity result of [1][Lemma 11.1] that, for $\pi: X \rightarrow$ Spec $k$ the structure morphism, the natural map

$$
\pi^{*} \Gamma_{\text {Spec } k}(a)_{\text {ét }} \otimes^{L} \mathbb{Z} / m \rightarrow \Gamma_{X}(a)_{\text {ét }} \otimes^{L} \mathbb{Z} / m
$$

is a quasi-isomorphism. It follows from the Suslin-Voevodsky theorem relating Suslin cohomology and étale cohomology [18][Corollary 7.8], together with Suslin's comparison of Suslin homology and motivic cohomology [17], that the cycle class map induces a quasi-isomorphism

$$
\Gamma_{\text {Spec } k}(a)_{\text {ét }} \otimes^{L} \mathbb{Z} / m \rightarrow \mu_{m}^{\otimes a}
$$

for $k$ algebraically closed of characteristic zero. This gives us the natural quasiisomorphism of étale sheaves on $X$

$$
\mu_{m}^{\otimes a} \rightarrow \Gamma_{X}(a)_{\text {ét }} \otimes^{L} \mathbb{Z} / m .
$$

The case of $k$ separably closed of characteristic $p>0$ follows as above by using de Jong's resolution of singularities [8] to extend the results of [18] to positive characteristic (see e.g. [9] for some further details).

\section{REFERENCES}

[1] Bloch, S. Algebraic cycles and higher K-theory, Adv. in Math 61 (1986), 267-304

[2] Bloch, S. The moving lemma for higher Chow groups J. Algebraic Geom. 3 (1994), no. 3, 537-568.

[3] Bloch, S., Kato, K. p-adic étale cohomology, Inst. Hautes Études Sci. Publ. Math. 63 (1986), 107-152.

[4] Bloch, S., Ogus, A. Gersten's conjecture and the homology of schemes, Ann. Sci. École Norm. Sup. (4) 7 (1974), 181-201 (1975).

[5] Colliot-Thélène, J.-L., Hoobler, R. T., Kahn, B. The Bloch-Ogus-Gabber theorem, Algebraic $K$-theory (Toronto, ON, 1996), 31-94, Fields Inst. Commun., 16, Amer. Math. Soc., Providence, RI, 1997.

[6] Gabber, O. Affine analog of the proper base change theorem, Israel J. Math. 87 (1994), no. $1-3,325-335$.

[7] Geisser, T. and Levine, M. The K-theory of fields in characteristic $p$, preprint, 1998.

[8] de Jong, A. J. Smoothness, semi-stability and alterations, Inst. Hautes Études Sci. Publ. Math. 83 (1996), 51-93.

[9] Levine, M. Homology of algebraic varieties: An introduction to the works of Suslin and Voevodsky, Bull. AMS 34 (1997) no. 3, 293-312.

[10] Levine, M. Relative Milnor K-theory, K-theory 6 (1992), 113-175.

[11] Levine, M. Mixed Motives, to appear, AMS Monographs and Surveys.

[12] Lichtenbaum, Stephen. Motivic complexes. Motives (Seattle, WA, 1991), 303-313, Proc. Sympos. Pure Math., 55, Part 1, Amer. Math. Soc., Providence, RI, 1994.

[13] Merkurjev, A., Suslin, A. K-cohomology of Severi-Brauer varieties and the norm residue homomorphism, Izv. Akad. Nauk SSSR Ser. Mat. 46 (1982), no. 5, 1011-1046, 1135-1136.

[14] Milne, J. Étale cohomology Princ. Univ. Press 1980.

[15] Sherman, C. K-cohomology of regular schemes, Com.Alg. 7 (1979), 999-1027.

[16] Strano, R. On the étale cohomology of Hensel rings, Comm. Algebra 12 (1984), no. 17-18, 2195-2211.

[17] Suslin, A. Higher Chow groups of affine varieties and étale cohomology, preprint (1995).

[18] Suslin, A. and Voevodsky, V. Singular homolgoy of abstract algebraic varieties, Invent. Math. 123 (1996) 61-94. 
[19] Suslin, A. and Voevodsky, V. Bloch-Kato conjecture and motivic cohomology with finite coefficients, preprint (1995).

[20] Voevodsky, V., Triangulated categories of motives over a field, preprint (1995).

[21] Voevodsky, V., The Milnor Conjecture, preprint.

University of Illinois, 1409 W. Green St., Urbana, IL, USA, and Department of Mathematics, Northeastern University, Boston, MA 02115, USA 